\title{
Properties of convergence of a class of iterative processes generated by sequences of self-mappings with applications to switched dynamic systems
}

Manuel De la Sen ${ }^{*}$ and Asier Ibeas ${ }^{2}$

\section{"Correspondence:}

manuel.delasen@ehu.es

1 Institute of Research and

Development of Processes,

University of the Basque Country,

Campus of Leioa (Bizkaia), Barrio

Sarriena, P.O. Box 644, Bilbao, 48940,

Spain

Full list of author information is

available at the end of the article

\begin{abstract}
This article investigates the convergence properties of iterative processes involving sequences of self-mappings of metric or Banach spaces. Such sequences are built from a set of primary self-mappings which are either expansive or non-expansive self-mappings and some of the non-expansive ones can be contractive including the case of strict contractions. The sequences are built subject to switching laws which select each active self-mapping on a certain activation interval in such a way that essential properties of boundedness and convergence of distances and iterated sequences are guaranteed. Applications to the important problem of stability of dynamic switched systems are also given.
\end{abstract}

Keywords: expansive; non-expansive; contractive and strictly contractive self-mappings; switched dynamic systems; convergence; fixed point; stability

\section{Introduction}

The problems of boundedness and convergence of sequences of iterative schemes are very important in numerical analysis and the numerical implementation of discrete schemes. See [1-5] and references therein. In particular, [1] describes in detail and with rigor the associated problems linked to the theory of fixed points in various types of spaces like metric spaces, complete and compact metric spaces and Banach spaces. This book also contains, discusses and compares results of a number of relevant background references on the subject. In other papers, related problems are focused from a computational point of view including the acceleration of convergence using modified numerical methods like Aitken's delta-squared methods or Steffensen's method, [2-5]. On the other hand, there is also a rich background in theory and applications of fixed point theory related to non-expansive, contractive, weakly contractive and strictly contractive mappings as well as related to their counterparts in the framework of common fixed points and coincidence points for several mappings and in the framework of multivalued functions. A (non-exhaustive) list of recent related references is given including new results and a discussion of previous background ones. See, for instance, $[1,6-25]$, and references therein. Many efforts are also devoted to the formulation of extensions of the above problems to the study of existence and uniqueness of best proximity points in cyclic self-mappings, $[8,13,14,16-21]$, to that of proximal

\section{Springer}

@2014 De la Sen and lbeas; licensee Springer. This is an Open Access article distributed under the terms of the Creative Commons Attribution License (http://creativecommons.org/licenses/by/2.0), which permits unrestricted use, distribution, and reproduction in any medium, provided the original work is properly cited. 
contractions, $[13,14]$ and to the characterization of approximate fixed and coincidence points, [22, 23]. Direct applications of fixed point theory to the study of the stability of dynamic systems including the property of ultimate boundedness for the trajectory solutions having mixed non-expansive and expansive properties through time or being subject to impulsive controls have been given in [21] and [24, 25]. Some recent studies of best proximity points of weak $\phi$-contractions in ordered metric spaces have been performed in [26]. On the other hand, the existence of best proximity points for 2-cyclic operators in uniformly convex Banach spaces is investigated in [27]. Finally, in [28], it has been proved that some previous fixed point results and some recently announced best proximity results are equivalent.

This paper is focused on the study of boundedness and convergence of sequences of distances and iterated points and the characterization of fixed points of a class of composite self-maps in metric spaces. Such maps are built with combinations of sets of elementary self-maps which can be expansive or non-expansive and the last ones can be contractive (including the case of strict contractions). The composite maps are defined by switching rules which select some self-map (the 'active' self-map) on a certain interval of definition of the running index of the sequence of iterates being built. The above mentioned properties concerning the sequences of iterates being generated from the given initial points are investigated under particular constraints for the switching rule. Note, on the other hand, that the properties of controllability and stability of differential-difference and the various kinds of dynamic systems are of a wide interest in theory and applications [25, 29-52]. See, for instance, related problems associated with continuous-time, discrete-time, digital, and hybrid systems and those involving delayed dynamics [38-42] and [31, 34-37, 44-46], sampled-data systems under constant, non-uniform, and/or multirate sampling and switched systems $[34,36,45,46]$. In this context, this paper also includes an application of the developed theoretical framework to the stability of (in general) nonlinear switched dynamic systems. The composite self-map generating the trajectory solution sequence from initial conditions is defined with sets of elementary self-maps being associated with distinct active parameterizations which are switched through time by switching rules which guarantee the fulfilment of the suitable properties.

\section{Problem statement and main results}

Consider a sequence $\left\{T_{\sigma(n)}\right\}$ of self-mappings from $X$ to $X$ where $(X, d)$ is a metric space endowed with a metric $d: X \times X \rightarrow \mathbf{R}_{0+}$ and an iterative process

$$
x_{n+1}=T_{\sigma(n)} x_{n}, \quad x_{0} \in X, \forall n \in \mathbf{Z}_{0+} .
$$

The subsequent nomenclature is used:

(1) The function $\sigma: \mathbf{Z}_{0_{+}} \rightarrow \bar{q} \subseteq \mathbf{Z}_{+}$is the so-called switching law where $\bar{q}=\{1,2, \ldots, q\}$ if $q \in \mathbf{Z}_{+}$is finite and $\bar{q} \equiv \mathbf{Z}_{+}$, otherwise, i.e. $q=\operatorname{card}(\bar{q})=\chi_{0}$ (i.e. the infinity cardinal of a countable set).

(2) $q$ is the set of distinct parameterizations of the sequence $S T=\left\{T_{\sigma(n)}\right\}$ of selfmappings on $X$ in the sense that such a sequence contains a finite or infinite set $S T^{*}=$ $\left\{T_{1}, T_{2}, \ldots, T_{q}\right\}$ of distinct self-mappings.

(3) $q$ is the disjoint union of the sets $\bar{q}=q_{s c} \cup q_{0 c} \cup q_{n e} \cup q_{e}$, where $q_{s c}, q_{0 c}$, $q_{n e}$, and $q_{e}$ are, respectively, the indexing sets of strict contractions, contractive self-mappings which 
are not strict contractions, non-expansive self-mappings which are not contractive, and a class of expansive self-mappings which fulfil a specific expanding condition according to

$$
\begin{aligned}
& d\left(T_{i} x, T_{i} y\right) \leq K_{i} d(x, y), \quad \forall x, y \in X \text { with } K_{i} \in(0,1) \text { if } i \in q_{s c}, \\
& d\left(T_{i} x, T_{i} y\right)<d(x, y)=K_{i} d(x, y), \quad \forall x, y(\neq x) \in X \text { with } K_{i}=1 \text { if } i \in q_{0 c}, \\
& d\left(T_{i} x, T_{i} y\right)=K_{i} d(x, y)=d(x, y), \quad \forall x, y \in X \text { with } K_{i}=1 \text { if } i \in q_{n e}, \\
& K_{0 i} d(x, y) \leq d\left(T_{i} x, T_{i} y\right) \leq K_{i} d(x, y), \\
& \quad \forall x, y \in X \text { with } K_{0 i}, K_{i}\left(>K_{0 i}\right) \in(1, \infty) \text { if } i \in q_{e} .
\end{aligned}
$$

Note that $q_{c}=q_{s c} \cup q_{0 c}$ indexes the whole set of contractive self-mappings in the set $S T^{*}$.

(4) The composite mapping $S T: \mathbf{Z}_{0+} \times \mathbf{Z}_{+} \times X \rightarrow X, \forall n \in \mathbf{Z}_{0+}$ defined by

$$
\begin{aligned}
\hat{T}(n, n+m) & =T_{\sigma(n+m-1)} \hat{T}(n, n+m-1) \\
& =T_{\sigma(n+m-1)} T_{\sigma(n+m-2)} \cdots T_{\sigma(n)}, \quad \forall n \in \mathbf{Z}_{0+}, \forall m \in \mathbf{Z}_{+} ;
\end{aligned}
$$

in particular,

$$
\hat{T}_{n}=\hat{T}(0, n)=T_{\sigma(n)} \hat{T}_{n-1}=T_{\sigma(n)} T_{\sigma(n-1)} \cdots T_{\sigma(0)}
$$

with $\sigma(n) \in \bar{q}$, defines the sequence of iterations $\left\{x_{n}\right\}$ defined by

$$
x_{n+1}=\hat{T}(m, n+1) x_{m}=\hat{T}_{\hat{\sigma}(n, m+1)}=T_{\sigma(n)} x_{n}=\hat{T}_{n} x_{0}, \quad \forall x_{0} \in X, \forall m, n(\geq m) \in \mathbf{Z}_{0+},
$$

where $\hat{\sigma}(n, m+1)=\{\sigma(n), \sigma(n+1), \ldots, \sigma(m)\}$ is the sequence of configurations from $n$ to $m+1$ defined by the switching law $\sigma: \mathbf{Z}_{0+} \rightarrow \bar{q} \subseteq \mathbf{Z}_{+}$. One gets from recursion with (2.1)

$$
\begin{aligned}
d\left(\hat{T}_{n} x_{0}, \hat{T}_{n-1} x_{0}\right) & \leq K(0, n) d\left(x_{0}, T_{\sigma(0)} x_{0}\right) \\
& =K(0, n) d\left(x_{0}, T_{\sigma(0)} x_{0}\right), \quad \forall x_{0} \in X, \forall n \in \mathbf{Z}_{+}
\end{aligned}
$$

if $\sigma(j) \notin q_{0 c}, \forall j \in \bar{n} \cup\{0\}$, and

$$
\begin{aligned}
d\left(\hat{T}_{n+1} x_{0}, \hat{T}_{n} x_{0}\right) & \leq K(0, n) K_{0 c}(0, n) d\left(x_{0}, T_{\sigma(0)} x_{0}\right) \\
& <K(0, n) d\left(x_{0}, T_{\sigma(0)} x_{0}\right), \quad \forall x_{0} \in X,
\end{aligned}
$$

if there is some $j \in \bar{n} \cup\{0\}$ such that $\sigma(j) \in q_{0 c}$, where

$$
\begin{aligned}
& K(0, n)=\prod_{j=0}^{n-1}\left[K_{\sigma(j)}\right], \\
& K_{0 c}(0, n)=\prod_{j\left(\in \sigma_{0 c}\right)=0}^{n-1}\left[\frac{d\left(T_{\sigma(j+1)} x_{j+1}, T_{\sigma(j+2)} x_{j}\right)}{d\left(T_{\sigma(j)} x_{j}, T_{\sigma(j+1)} x_{j+1}\right)}\right](<1), \quad \forall n \in \mathbf{Z}_{0+} .
\end{aligned}
$$

The following simple example illustrates how the iterative process can work in a real situation. 
Example 2.1 Consider the simple scalar discrete equation (2.1) with $x_{n+1}=T_{\sigma(n)} x_{n}=a_{n} x_{n}$ with $x_{0} \in \mathbf{R}, \forall n \in \mathbf{Z}_{0+}$ under the Euclidean metric. The sequence $\left\{T_{\sigma(n)}=a_{n}\right\}$ is such that $T_{\sigma(n)} \in\left\{T_{1}, T_{2}, T_{3}\right\}, \forall n \in \mathbf{Z}_{0+}$, where $T_{1}=\{z \in \mathbf{R}: z \leq \alpha\}, T_{2}=\{1\}$, and $T_{3}=\{z \in \mathbf{R}: z \geq \beta\}$ for some given real constants $-1<\alpha<1$ and $\beta>1$. It is clear that the self-mapping $T_{1}$ on $\mathbf{R}$ is a strict contraction with a contractive constant $K_{1}=|\alpha|<1$, the self-mapping $T_{2}$ on $\mathbf{R}$ is non-expansive with constant $K_{2}=1$, and the self-mapping $T_{3}$ on $\mathbf{R}$ is expansive with constants $K_{03}=1$ and $K_{3}=\beta>1$. Note that the set of fixed points of $T_{i}$ reduces to $\{0\}$, that is, $\operatorname{Fix}\left(T_{i}\right)=\{0\}$ for $i \in \bar{q}\{0\}$ is the unique fixed, and equilibrium, point of $T_{1}$ and $T_{3}$ while $\operatorname{Fix}\left(T_{2}\right)=\mathbf{R}$ with the whole $\mathbf{R}$ being an equilibrium set of $T_{2}$. Note also that $\{0\}$ is a stable equilibrium point of $T_{1}$, while it is an unstable equilibrium point of $T_{3}$.

The switching law is $\sigma: \mathbf{Z}_{0+} \rightarrow \bar{q}=\{1,2,3\} \subseteq \mathbf{Z}_{+}$. If $\sigma(n)=i(\in \bar{q}), \forall n \in\left[n_{1}, n_{2}\right) \cap \mathbf{Z}_{0^{+}}$, it is said that the $i$ th configuration of (2.1) is active in the interval $\left[n_{1}, n_{2}\right)$. If $\sigma\left(n_{2}\right)=j(\in \bar{q}) \neq i$, then it is said that $n_{2}$ is a switching sample of (2.1) since the active configuration becomes modified with such a sample.

Lemma 2.2 Consider the iterative process (2.1) built with a sequence $\left\{T_{\sigma(n)}\right\}$ of selfmappings from $X$ to $X$ such that $(X, d)$ is a metric space. Assume a given switching law $\sigma: \mathbf{Z}_{0_{+}} \rightarrow \bar{q} \subseteq \mathbf{Z}_{+}$such that there are a strictly increasing testing sequence of integers $S=S(\sigma)=\left\{n_{k}\right\}$, which may be non-unique, with $n_{0}=0$, and an associate sequence of positive real numbers $S_{\rho}=S_{\rho}(S)=\left\{\rho_{k}\right\}$ verifying $n_{k+1}-n_{k} \leq \mu<+\infty$ and $K\left(n_{k}, n_{k+1}\right)=$ $\prod_{j=n_{k}}^{n_{k+1}-1}\left[K_{\sigma(j)}\right] \leq \rho_{k}, \forall k \in \mathbf{Z}_{0+}$, where $\mu=\mu(S)$. Then the metric has the following properties:

(i) If $\rho_{k}<1, \forall k \in \mathbf{Z}_{0+}$ then $d\left(x_{n+1}, x_{n}\right) \rightarrow 0$ as $n \rightarrow \infty, \forall x_{0} \in X$, and $d\left(x_{n_{k+1}}, x_{n_{k}}\right) \leq$ $\left(\sum_{i=0}^{\mu-1} M_{0}^{i}\right) \rho_{k} d\left(x_{n_{k}}, x_{n_{k-1}}\right) \forall k \in \mathbf{Z}_{+}, \forall x_{0} \in X, d\left(x_{n_{k}}, x_{n_{k+1}}\right) \rightarrow 0$ as $k \rightarrow \infty$ and $d\left(x_{n}, x_{n+1}\right) \rightarrow$ 0 as $n \rightarrow \infty, \forall x_{0} \in X$, where $M_{0}=\max _{i \in \bar{q}}\left(K_{i}\right)$.

(ii) If the switching law $\sigma: \mathbf{Z}_{0+} \rightarrow \bar{q} \subseteq \mathbf{Z}_{+}$is such that $\rho_{k} \leq \rho /\left(\sum_{i=0}^{\mu} M_{0}^{i}\right), \forall k \in \mathbf{Z}_{0+}$, for some given real constant $\rho \in(0,1)$ then all the sequence of composite self-mappings defined by $\hat{T}\left(n_{k}, n_{k+1}\right)=T_{\sigma\left(n_{k+1}-1\right)} \cdots \hat{T}_{\sigma\left(n_{k}\right)}$ on $X$ are strict contractions of contractive constant $\rho$.

(iii) If $\rho_{k}>1, \forall k \in \mathbf{Z}_{0+}$ then $d\left(x_{n+1}, x_{n}\right) \rightarrow+\infty$ as $n \rightarrow \infty$ for any given $x_{0} \notin \operatorname{Fix}\left(T_{\sigma(0)}\right)$ in $X$.

(iv) Assume that $\rho_{k} \leq \bar{\rho}<1, \forall k \in \mathbf{Z}_{0+}$ for $n_{k} \in S$ and $\rho_{k} \in S_{\rho}, \forall k \in \mathbf{Z}_{0+}$ and the existence of the limits $\lim _{k \rightarrow \infty}\left(n_{k+1}-n_{k}\right)=\mu^{*} \leq \mu<+\infty$ and $\hat{T}\left(n_{k}, n_{k+1}\right) \rightarrow \hat{T}^{*}\left(0, \mu^{*}\right)$ uniformly in $X$ as $k \rightarrow \infty$, that is, $\lim _{k \rightarrow \infty} \sup \left\|\hat{T}\left(n_{k}, n_{k+1}\right) x-\hat{T}^{*}\left(0, \mu^{*}\right) x\right\|=0, \forall x \in X$, where $\mu^{*}=\mu^{*}(S)$. Then the self-mapping $\hat{T}^{*}\left(0, \mu^{*}\right)$ on $X$ is a strict contraction and, for any given $N \in \mathbf{Z}_{0+}$, there is a real constant $\delta=\delta(N)$ such that $\{\delta(N)\} \rightarrow 0$ such that one has for all $k, N \in \mathbf{Z}_{0+}$

$$
\begin{aligned}
& d\left(x_{k+N+1}, x_{k+N}\right) \leq \bar{\rho}^{k} d\left(x_{n_{0}}, x_{n_{1}}\right)+\frac{1-\bar{\rho}^{k}}{1-\bar{\rho}} \max _{i \in \mathbf{Z}_{0+}}\left(\delta_{n_{i}}\right), \\
& \lim _{k, N \rightarrow \infty} d\left(x_{n_{k+N+1}}, x_{n_{k+N}}\right)=0 .
\end{aligned}
$$

Proof Note that $n_{0}=0$ so that for any $n_{k} \in S$ and $k \in \mathbf{Z}_{0+}$

$$
\begin{aligned}
d\left(x_{n_{k+1}+1}, x_{n_{k+1}}\right) & \leq K\left(n_{k}, n_{k+1}\right) d\left(x_{n_{k}}, x_{n_{k}+1}\right) \leq \rho_{k} d\left(x_{n_{k}}, x_{n_{k}+1}\right), \quad \forall k \in \mathbf{Z}_{0+} \\
& \leq \cdots \leq\left(\prod_{i=0}^{k}\left[\rho_{i}\right]\right) d\left(T_{\sigma(0)} x_{0}, x_{0}\right)<d\left(T_{\sigma(0)} x_{0}, x_{0}\right),
\end{aligned}
$$




$$
\begin{aligned}
d\left(x_{n_{k+1}+j+1}, x_{n_{k+1}+j}\right) \leq & \left(\prod_{i=n_{k}+1}^{n_{k+1}+j}\left[K_{\sigma(i)}\right]\right)\left(\prod_{i=0}^{k}\left[\rho_{i}\right]\right) d\left(T_{\sigma(0)} x_{0}, x_{0}\right) \\
\leq & M\left(n_{k}, j\right)\left(\prod_{i=0}^{k}\left[\rho_{i}\right]\right) d\left(T_{\sigma(0)} x_{0}, x_{0}\right) \\
\leq & M\left(\prod_{i=0}^{k}\left[\rho_{i}\right]\right) d\left(T_{\sigma(0)} x_{0}, x_{0}\right)<M\left(\prod_{i=0}^{k}\left[\rho_{i}\right]\right) d\left(T_{\sigma(0)} x_{0}, x_{0}\right), \\
& \forall k \in \mathbf{Z}_{0+}, \forall j \in \overline{n_{k}-n_{k+1}-1} \cup\{0\}
\end{aligned}
$$

(the last inequalities of (2.11)-(2.12) standing if and only if the respective left-hand-sides are nonzero) for some set of real bounded sequences $\left\{M\left(n_{k}, j\right)\right\}, j \in \overline{n_{k+2}-n_{k+1}}$ which satisfy $M\left(n_{k}, j\right) \leq M=M_{0} \max \left(1, M_{0}^{\mu-1}\right)<+\infty$ for some $M \in \mathbf{R}_{+}$and $j \in \overline{n_{k+2}-n_{k+1}}$, since $\max _{k \in \mathbf{Z}_{0+}}\left(n_{k+1}-n_{k}\right) \leq \mu<+\infty, \forall k \in \mathbf{Z}_{0+}$ and $K_{i} \in(1, \infty), \forall i \in \bar{q}$. There is $k_{0} \in \mathbf{Z}_{0+}$ such that $\left(\prod_{i=0}^{k}\left[\rho_{i}\right]\right)<1 / M$, since $\rho_{i}<1, \forall i \in \mathbf{Z}_{0+}$, then one gets from (2.8)

$$
d\left(x_{n_{k+1}+j+1}, x_{n_{k+1}+j}\right)<d\left(T_{\sigma(0)} x_{0}, x_{0}\right), \quad \forall k\left(\geq k_{0}\right) \in \mathbf{Z}_{0+} .
$$

Also, since $\rho_{k}<1, \forall k \in \mathbf{Z}_{0+}$, one gets from (2.11)-(2.12)

$$
\lim _{k \rightarrow \infty} d\left(x_{n_{k}+j+1}, x_{n_{k}+j}\right)=0, \quad \forall j \in \overline{n_{k+1}-n_{k}-1} \cup\{0\}
$$

Since (2.14) involves any limit distance in-between any two adjacent elements of the solution sequence $\left\{x_{n}\right\}$ of (2.1) for any given $x_{0} \in X$, one gets $\lim _{n \rightarrow \infty} d\left(x_{n+1}, x_{n}\right)=0$. On the other hand, one gets from (2.11) and the triangle inequality

$$
d\left(x_{n_{k+1}}, x_{n_{k}}\right) \leq \sum_{i=1}^{n_{k+1}-n_{k}-1} d\left(x_{n_{k}+i}, x_{n_{k}+i+1}\right) \leq \rho_{k}\left(\sum_{i=0}^{\mu-1} M_{0}^{i}\right) d\left(x_{n_{k}}, x_{n_{k}+1}\right), \quad \forall k \in \mathbf{Z}_{0+}
$$

so that $d\left(x_{n_{k+1}}, x_{n_{k}}\right) \leq\left(\sum_{i=0}^{\mu-1} M_{0}^{i}\right) \rho_{k} d\left(x_{n_{k}}, x_{n_{k-1}}\right), \forall k \in \mathbf{Z}_{+}$and $d\left(x_{n_{k}}, x_{n_{k+1}}\right) \rightarrow 0$ as $k \rightarrow \infty$ from the triangle inequality, $d\left(x_{n}, x_{n+1}\right) \rightarrow 0$ as $n \rightarrow \infty$ and $\max _{k \in \mathbf{Z}_{0+}}\left(n_{k+1}-n_{k}\right) \leq \mu<+\infty$. Hence, property (i) follows. Property (ii) is proven by noting that if $\rho_{k} \leq \rho /\left(\sum_{i=0}^{\mu-1} M_{0}^{i}\right)$, $\forall k \in \mathbf{Z}_{0+}$, for some real $\rho \in(0,1)$ then

$$
d\left(x_{n_{k+1}}, x_{n_{k}}\right) \leq \rho d\left(x_{n_{k}}, x_{n_{k-1}}\right) \leq \rho^{2} d\left(x_{n_{k-1}}, x_{n_{k-2}}\right) \leq \cdots \leq \rho^{k} d\left(x_{n_{0}}, x_{n_{1}}\right) .
$$

Property (iii) is proven from the fact that expansive mappings of the switching law fulfil

$$
d(x, y)<K_{0 i} d(x, y) \leq d\left(T_{i} x, T_{i} y\right), \quad \forall x, y \in X \text { with } K_{0 i}>1 \text { if } i \in q_{e}
$$

and it follows from the assumptions that there is an infinite sequence $S=\left\{n_{k}\right\}$ such that $T_{\sigma\left(n_{k}\right)}$ on $X$ are non-expansive self-mappings. Then $d\left(x_{n_{k+1}}, x_{n_{k+1}+1}\right) \geq\left(\prod_{i=0}^{k}\left[\rho_{i}\right]\right) d\left(T_{\sigma(0)} x_{0}\right.$, $\left.x_{0}\right), \forall k \in \mathbf{Z}_{0+}$ so that $\lim _{k \rightarrow \infty} d\left(x_{n_{k+1}}, x_{n_{k+1}+1}\right)=+\infty$ if $x_{0} \notin \operatorname{Fix}\left(T_{\sigma(0)}\right)$. Thus, $\lim _{k \rightarrow \infty} d\left(x_{n_{k+1}+j}\right.$, $\left.x_{n_{k+1}+j+1}\right)=+\infty$ for all $j=0,1, \ldots, n_{k+2}-n_{k+1}-1$ since $n_{k+2}-n_{k+1} \leq \mu$. Otherwise, it would be impossible with the triangle inequality of the metric to be bounded over a sum of a finite number of distances as $k \rightarrow \infty$ of which one is unbounded as $k \rightarrow \infty$. Hence, 
property (iii) follows. To prove property (iv), note that $\delta_{n_{k}}=d\left(\hat{T}\left(n_{k-1}, n_{k}\right) x, \hat{T}\left(n_{k}, n_{k+1}\right) x\right)$ leads to $\delta_{n_{k}} \rightarrow 0$ as $k \rightarrow \infty$, so that $\left\{\delta_{n_{k}}\right\}$ is bounded since $\left(n_{k+1}-n_{k}\right) \rightarrow \mu^{*}$ as $k \rightarrow \infty$ and $\hat{T}\left(n_{k}, n_{k+1}\right) \rightarrow \hat{T}^{*}\left(0, \mu^{*}\right)$ uniformly in $X$ as $k \rightarrow \infty$. Then $\rho_{k} \leq \bar{\rho}<1, \forall k \in \mathbf{Z}_{0+}$ for $n_{k} \in S$ and $\rho_{k} \in S_{\rho}, \forall k \in \mathbf{Z}_{0+}$ and the existence of the $\operatorname{limits} \lim _{k \rightarrow \infty}\left(n_{k+1}-n_{k}\right)=$ $\mu^{*} \leq \mu<+\infty, \lim _{k \rightarrow \infty} \rho_{k}=\rho^{*} \leq \bar{\rho}<1$ and $\hat{T}\left(n_{k}, n_{k+1}\right) \rightarrow \hat{T}^{*}\left(0, \mu^{*}\right)$ uniformly in $X$ as $k \rightarrow \infty$ imply that $\hat{T}^{*}\left(0, \mu^{*}\right)$ is a strict contraction on $X$ and, furthermore, since $\left[d\left(\hat{T}\left(n_{k-1}, n_{k}\right) x_{n_{k}}, \hat{T}\left(n_{k}, n_{k+1}\right) x_{n_{k}}\right)-d\left(\hat{T}\left(0, \mu^{*}\right) x_{n_{k}}, \hat{T}\left(0, \mu^{*}\right) x_{n_{k}}\right)\right] \rightarrow 0$ as $k \rightarrow \infty$, one gets

$$
\begin{aligned}
d\left(x_{n_{k+1}}, x_{n_{k}}\right) & =d\left(\hat{T}\left(n_{k}, n_{k+1}\right) x_{n_{k}}, \hat{T}\left(n_{k-1}, n_{k}\right) x_{n_{k-1}}\right) \\
& \leq d\left(\hat{T}\left(n_{k-1}, n_{k}\right) x_{n_{k-1}}, \hat{T}\left(n_{k-1}, n_{k}\right) x_{n_{k}}\right)+d\left(\hat{T}\left(n_{k-1}, n_{k}\right) x_{n_{k}}, \hat{T}\left(n_{k}, n_{k+1}\right) x_{n_{k}}\right) \\
& \leq K\left(n_{k-1}, n_{k}\right) d\left(x_{n_{k-1}}, x_{n_{k}}\right)+\delta_{n_{k}} \\
& \leq\left(\prod_{i=1}^{k}\left[\rho_{i}\right]\right) d\left(x_{n_{0}}, x_{n_{1}}\right)+\sum_{i=0}^{k} \prod_{j=i+1}^{k}\left[\rho_{i}\right] \delta_{n_{i}} \\
& \leq \bar{\rho}^{k} d\left(x_{n_{0}}, x_{n_{1}}\right)+\frac{1-\bar{\rho}^{k}}{1-\bar{\rho}} \max _{i \in \mathbf{Z}_{0+}}\left(\delta_{n_{i}}\right)
\end{aligned}
$$

and then (2.9) holds. Also, (2.10) follows from (2.9), since $\lim _{N \rightarrow \infty} \max _{i(\geq N) \in \mathbf{Z}_{0+}}\left(\delta_{n_{i}}\right)=0$ for any given $x_{0} \in X$ and $\bar{\rho}^{n_{k+N}-n_{N}} \rightarrow 0$ as $k, N \rightarrow \infty$, and then

$$
\begin{aligned}
0 & \leq \lim \sup _{k \rightarrow \infty} d\left(x_{n_{k+1}}, x_{n_{k+2}}\right) \\
& \leq \lim \sup _{k \rightarrow \infty}\left(d\left(x_{n_{k+1}}, x_{n_{k+2}}\right)-\bar{\rho}^{\mu *} d\left(x_{n_{k}}, x_{n_{k+1}}\right)-\frac{1-\bar{\rho}^{\mu *}}{1-\bar{\rho}} \max _{i \in \mathbf{Z}_{0+}}\left(\delta_{n_{i}}\right)\right) \\
& \leq \lim \sup _{k \rightarrow \infty}\left(d\left(x_{n_{k+1}}, x_{n_{k+2}}\right)-K\left(0, \mu^{*}\right) d\left(x_{n_{k}}, x_{n_{k+1}}\right)-\frac{1-\bar{\rho}^{\mu *}}{1-\bar{\rho}} \max _{i \in \mathbf{Z}_{0+}}\left(\delta_{n_{i}}\right)\right) \leq 0
\end{aligned}
$$

so that $\lim _{k \rightarrow \infty} d\left(x_{n_{k+1}}, x_{n_{k+2}}\right)$ exists and then, since $\lim _{k \rightarrow \infty}\left(n_{k+1}-n_{k}\right)=\mu^{*} \leq \mu<+\infty$ and $\hat{T}\left(n_{k}, n_{k+1}\right) \rightarrow \hat{T}^{*}\left(0, \mu^{*}\right)$ uniformly in $X$ as $k \rightarrow \infty$ and (2.14)-(2.15) hold, one gets for any nonnegative integer $j \leq \mu^{*}$

$$
\begin{aligned}
\lim _{k \rightarrow \infty} d\left(x_{n_{k}}, \hat{T}\left(n_{k}, n_{k+1}\right) x_{n_{k}}\right) & =\lim _{k \rightarrow \infty} d\left(x_{n_{k}}, \hat{T}^{*}\left(0, \mu^{*}\right) x_{n_{k}}\right) \\
& =\lim _{k \rightarrow \infty} d\left(x_{n_{k}+j}, \hat{T}\left(n_{k+j}, n_{k+j+1}\right) x_{n_{k}}\right) \\
& =\lim _{k \rightarrow \infty} d\left(x_{n_{k}+j}, \hat{T}^{*}\left(0, \mu^{*}\right) x_{n_{k}+j}\right) \\
& =\lim _{k \rightarrow \infty} d\left(x_{n_{k}+\mu^{*}+j}, \hat{T}^{*}\left(0, \mu^{*}\right) x_{n_{k}+j}\right)=0
\end{aligned}
$$

and property (iv) has been proven.

Remark 2.3 Note that the testing sequence $S=\left\{n_{k}\right\}$ of the given switching law in Lemma 2.2 is not necessarily associated with a set of strict contractions although the composite $K\left(n_{k}, n_{k+1}\right)$, for $n_{k} \in S$, defines a composite strict contraction of non-necessarily strictly contractive self-mappings. The composite sequences $K\left(n, n_{k}\right)$ for $n \in\left[n_{k}+1, n_{k+1}\right)$, where $n_{k} \in S$, are not necessarily associated with a composite strict contraction. Note also that the testing sequence $S=\left\{n_{k}\right\}$ is not unique for a given switching law $\sigma: \mathbf{Z}_{0+} \rightarrow \bar{q} \subseteq \mathbf{Z}_{+}$ 
since the only requirement is that it be strictly increasing with a maximum prescribed, but arbitrary, separation in-between any two adjacent elements of such a sequence.

Remark 2.4 Lemma 2.2 can be fulfilled, in general, by non-unique sequences $S=\left\{n_{k}\right\}$ of a given switching law in Lemma 2.2 as well as non-unique associated $\left\{\rho_{k}\right\}, \mu$ and $\mu^{*}$. A typical case occurs when the switching law consists of strict contractions or converges to a sequence of strict contractions. Those ones can be grouped individually in the limit $\hat{T}^{*}\left(0, \mu^{*}\right)$ or this one may be a composite self-mapping of strict limit contractions so that the next result follows.

Theorem 2.5 Assume an iterative procedure (2.1) built from a given switching law $\sigma: \mathbf{Z}_{0+} \rightarrow \bar{q} \subseteq \mathbf{Z}_{+}$. If the self-mapping $\hat{T}^{*}\left(0, \mu^{*}\right)$ on $X$ is a strict contraction such that $\hat{T}\left(n_{k}, n_{k+1}\right) \rightarrow \hat{T}^{*}\left(0, \mu^{*}\right)$ uniformly in $X$ as $k \rightarrow \infty$ with the strictly increasing testing sequence $S=\left\{n_{k}\right\}$ being subject to $\max _{k \in \mathbf{Z}_{0+}}\left(n_{k+1}-n_{k}\right) \leq \mu<+\infty$ and $\lim _{k \rightarrow \infty}\left(n_{k+1}-n_{k}\right)=$ $\mu^{*} \leq \mu$. Then the following properties hold:

(i) There is a (in general, non-unique) decomposition of the composite $\hat{T}^{*}\left(0, \mu^{*}\right): X \rightarrow X$ in a maximum number of $p \in\left[1, \mu^{*}\right]$ strict contractions $\hat{T}_{i}^{*}\left(\bar{\mu}_{i-1}^{*}, \bar{\mu}_{i}^{*}\right): X \rightarrow X, \forall i \in \bar{p}$, with $\mu_{0}^{*}=\bar{\mu}_{0}^{*}=0$ for the testing sequence $S=\left\{n_{k}\right\}$, such that $\mu^{*}=\bar{\mu}^{*}=\sum_{i=1}^{p} \mu_{i}^{*}$ so that

$$
\hat{T}^{*}\left(0, \mu^{*}\right)=\hat{T}^{*}\left(0, \bar{\mu}^{*}\right)=\hat{T}_{p}^{*}\left(\bar{\mu}_{p-1}^{*}, \bar{\mu}_{p}^{*}\right) \cdot \hat{T}_{p-1}^{*}\left(\bar{\mu}_{p-2}^{*}, \bar{\mu}_{p-1}^{*}\right) \cdot \ldots \cdot \hat{T}_{1}^{*}\left(0, \bar{\mu}_{1}^{*}\right),
$$

where $\bar{\mu}_{i}^{*}=\sum_{j=0}^{i} \mu_{i}^{*}, \forall i \in \bar{p}$.

(ii) The decomposition (2.20) in a maximum number of strict contractions is unique if and only if the positive integer numbers $\mu_{i}^{*}, \forall i \in \bar{p}$ such that $\mu^{*}=\sum_{i=1}^{p} \mu_{i}^{*}$ are unique. In particular, the decomposition (2.20) is unique if $\mu_{i}^{*}=1, \forall i \in \bar{p}$.

(iii) Assume that, furthermore, $\left(T_{\sigma(k n+j)}-T_{\sigma((k+1) n+j)}\right) \rightarrow 0$ uniformly in $X$ as $n \rightarrow \infty$ for some $k\left(\geq \mu^{*}\right) \in \mathbf{Z}_{0+}, \forall j \in \overline{k-1} \cup\{0\}$ so that $\{\sigma(k n+j)\} \rightarrow\left\{\sigma_{j}^{*}\right\}$ and $\left\{K_{\sigma(k n+j)}\right\} \rightarrow\left\{K_{j}^{*}\right\}, \forall j \in$ $\bar{\mu}^{*}$. Then the decomposition (2.20) is unique in a maximum number of strict contractions if and only if

$$
\begin{aligned}
& \mu_{i}^{*}=\min _{z \geq \mu_{i-1}^{*}}\left(z \in \mathbf{Z}_{+}:\left[\left(K_{z}^{*}<\frac{1}{\prod_{j=0}^{z-1}\left[K_{j}^{*}\right]}\right) \wedge\left(\prod_{j=z}^{\mu^{*}}\left[K_{j}^{*}\right]<1\right)\right]\right), \\
& \mu_{p}^{*}=\min _{z \geq \mu_{p-1}^{*}}\left(z \in \mathbf{Z}_{+}: K_{z}^{*}<\frac{1}{\prod_{j=0}^{z-1}\left[K_{j}^{*}\right]}\right),
\end{aligned}
$$

$\forall i \in \overline{p-1}$ since $\mu_{0}^{*}=\bar{\mu}_{0}^{*}=0$, where $p=\left(\max z \in \mathbf{Z}_{+}: \mu^{*}=\sum_{i=1}^{z} \mu_{i}^{*}\right)$. Then $\mu^{*}=\sum_{i=1}^{p} \mu_{i}^{*}$ with $\mu_{i}^{*}\left(\leq \mu^{*}\right) \in \mathbf{Z}_{+}, \forall i \in \bar{p}$ being unique as well.

Proof Since the switching law $\sigma: \mathbf{Z}_{0+} \rightarrow \bar{q} \subseteq \mathbf{Z}_{+}$is given, any strictly increasing sequence $S=\left\{n_{k}\right\}$ satisfying the constraints $\max _{k \in \mathbf{Z}_{0+}}\left(n_{k+1}-n_{k}\right) \leq \mu<+\infty, \lim _{k \rightarrow \infty}\left(n_{k+1}-n_{k}\right)=\mu^{*} \leq$ $\mu$ and $\hat{T}\left(n_{k}, n_{k+1}\right) \rightarrow \hat{T}^{*}\left(0, \mu^{*}\right)$ uniformly in $X$ as $k \rightarrow \infty$ for $n_{k}, n_{k+1} \in S$, with $\mu=\mu(S)$ and $\mu^{*}=\mu^{*}(S)$ being finite, imply that the composite-self-mapping $\hat{T}^{*}\left(0, \mu^{*}\right): X \rightarrow X$ is a composite self-mapping of at least one and at most $\mu^{*}$ strict contractions. So, the maximum number $p$ of strict limit contractions $p \in\left[1, \mu^{*}\right]$ in the composite $\hat{T}^{*}\left(0, \mu^{*}\right)$ for the sequence $\left\{T_{n_{k}}\right\}$ for $n_{k} \in S$ for a given sequence $S$ exists and satisfies (2.20). Property (i) follows. Uniqueness of the decomposition (2.20) holds if there is a unique $\mu_{i}^{*}, \forall i \in \bar{p}$ such 
that $\mu^{*}=\sum_{i=1}^{p} \mu_{i}^{*}$ and $\hat{T}_{i}^{*}\left(\bar{\mu}_{i-1}^{*}, \bar{\mu}_{i}^{*}\right): X \rightarrow X, \forall i \in \bar{p}$ are strict contractions. In particular, the decomposition (2.16) is trivially unique if $\mu_{i}^{*}=1 ; \forall i \in \bar{p}$ so that $p=\mu^{*}$. Property (ii) has been proven.

The proof of property (iii) is split into two parts as follows:

- $p=1$ and $\mu_{1}^{*}=\bar{\mu}_{1}^{*}=\mu^{*}$. Since (2.21a)-(2.21b) hold, $\mu_{1}^{*} \in \mathbf{Z}_{+}$, subject to $1 \leq \mu_{1}^{*} \leq \mu^{*}$ exists so that $\hat{T}\left(0, \mu_{1}^{*}\right)$ is a unique strict contraction on $X$. Thus, either $(2.21 \mathrm{~b})$ holds with $1 \leq \mu_{1}^{*}=\mu^{*}$ and the sufficiency part of the property is already proven for $p=1$ with a strict contraction $\hat{T}\left(0, \mu_{1}^{*}\right)=\hat{T}\left(0, \mu^{*}\right)$ on $X$ for $p=1$ or $p>1$ and (2.21a) holds for some integer $i \geq 1$. Necessity follows since if $p=1$ and $\hat{T}\left(0, \mu^{*}\right)$ is a strict contraction then $\hat{T}\left(0, \mu_{1}^{*}\right)=\hat{T}\left(0, \mu^{*}\right)$ for $\mu_{1}^{*}=\mu^{*}$. Uniqueness is trivial since there is a single self-mapping in the decomposition of the composite self-mapping $\hat{T}\left(0, \mu^{*}\right)$ on $X$.

$-p \geq 2$. The proof of sufficiency is proven by complete induction. Assume that (2.21a)(2.21b) hold so that $\mu_{1}^{*} \in \mathbf{Z}_{+}$, subject to $1 \leq \mu_{1}^{*} \leq \mu^{*}$, has to exist so that $\hat{T}\left(0, \mu_{1}^{*}\right)$ is a unique strict contraction on $X$ since $\mu^{*} \in \mathbf{Z}_{+}$exists such that $\hat{T}\left(0, \mu^{*}\right)$ exists, being a strict contraction on $X$. Set $\bar{\mu}_{0}^{*}=\mu_{0}^{*}=0$ and note that $\mu_{j}^{*}=\bar{\mu}_{j}^{*}-\bar{\mu}_{j-1}^{*}$ and $\bar{\mu}_{j}^{*}=\mu_{j}+\bar{\mu}_{j-1}^{*}=$ $\mu_{j}+\sum_{i=0}^{j-1} \mu_{i}, \forall j \in \bar{p}$, so that $\hat{T}\left(\bar{\mu}_{j-1}^{*}, \bar{\mu}_{j}^{*}\right)$, then $\mu_{j}^{*}=\bar{\mu}_{j}^{*}-\bar{\mu}_{j-1}^{*}$ is a unique strict contraction on $X$ for all $j(\leq i-1) \in \mathbf{Z}_{+}$and some $i(\leq p) \in \mathbf{Z}_{+}$such that the set of positive integers $\left\{\mu_{1}^{*}, \mu_{2}^{*}, \ldots, \mu_{i-1}^{*}\right\}$, subject to $\bar{\mu}_{i-1}^{*}=\sum_{j=1}^{i-1} \mu_{j}^{*}<\mu^{*}$, is unique. Then the composite $\hat{T}\left(0, \bar{\mu}_{i-1}^{*}\right)=\hat{T}\left(\bar{\mu}_{i-2}^{*}, \bar{\mu}_{i-1}^{*}\right) \cdot \ldots \cdot \hat{T}\left(0, \mu_{1}^{*}\right)$ is a unique strict contraction, with $\bar{\mu}_{i}^{*}=\sum_{j=0}^{i} \mu_{i}^{*}$, $\forall i \in \bar{p}$, and the positive integer $\mu_{i}^{*}$ is unique so that the set $\left\{\mu_{1}^{*}, \mu_{2}^{*}, \ldots, \mu_{i}^{*}\right\}$, subject to $\sum_{j=1}^{i} \mu_{j}^{*}<\mu^{*}$, is also unique and $\hat{T}\left(0, \mu_{j}^{*}\right)$, for all $j(\leq i) \in \mathbf{Z}_{+}$and $i(\leq p) \in \mathbf{Z}_{+}$, and then the composite $\hat{T}\left(0, \bar{\mu}_{i}^{*}\right)=\hat{T}\left(0, \mu_{i}^{*}\right) \cdot \ldots \hat{T}\left(0, \mu_{1}^{*}\right)$ are unique and strictly contractive on $X$. The proof follows by complete induction, $\forall i \in \bar{p}$ with the existence of a unique positive integer $p=p\left(\mu^{*}\right)=\left(\max z \in \mathbf{Z}_{+}: \mu^{*}=\sum_{i=1}^{z} \mu_{i}^{*}\right)$. Then $\mu^{*}=\sum_{i=1}^{p} \mu_{i}^{*}$ with $\mu_{i}^{*}\left(\leq \mu^{*}\right) \in \mathbf{Z}_{+}, \forall i \in \bar{p}$ being unique. Necessity follows since if (2.20) holds and (2.22) fails for some $i(\geq 2) \in \bar{p}$ for a given $p \geq 2$ then the factorization of the composite self-mapping $\hat{T}\left(0, \mu^{*}\right)$ on $X$, subject to $\mu^{*}=\sum_{i=1}^{p} \mu_{i}^{*}$, does not consist of strict contractions. Property (iii) has been proven.

A particular case of the decomposition of the composite self-mapping (2.20) is when such a decomposition becomes invariant and equal to

$$
\hat{T}^{*}\left(0, \mu^{*}\right)=\hat{T}_{p}^{*}\left(0, \mu_{p}^{*}\right) \cdot \hat{T}_{p-1}^{*}\left(0, \mu_{p-1}^{*}\right) \cdot \ldots \cdot \hat{T}_{1}^{*}\left(0, \mu_{1}^{*}\right)
$$

under the invariance identities $\hat{T}_{i}^{*}\left(0, \mu_{i}^{*}\right)=\hat{T}_{i}^{*}\left(\bar{\mu}_{i-1}^{*}, \bar{\mu}_{i}^{*}\right)$, implied by the identities $\mu_{i}=\bar{\mu}_{i}^{*}-$ $\bar{\mu}_{i-1}^{*}, \forall i \in \bar{p}$. If the collection of all the sequences $\hat{S}=\hat{S}(\sigma)=\left\{S=\left\{n_{k}\right\}\right\}$ for given $x_{0} \in X$ and $\sigma: \mathbf{Z}_{0+} \rightarrow \bar{q} \subseteq \mathbf{Z}_{+}$is considered under the given convergence hypotheses, it follows easily from the above reasoning that there is also a maximum number $\hat{p}$ of strict limit contractions $\hat{p} \in\left[1, \hat{\mu}^{*}\right]$, where

$$
\hat{\mu}^{*}=\max \left(\mu^{*}(S): S \in \hat{S}\right)=\max _{S \in \hat{S}}\left(\lim _{k \rightarrow \infty}\left(n_{k+1}-n_{k}\right): n_{k}, n_{k+1} \in S\right)=\max _{S \in \hat{S}} \mu^{*}(S)
$$

of the (in general, non-unique) decomposition:

$$
\hat{T}^{*}\left(0, \hat{\mu}^{*}\right)=\hat{T}_{\hat{p}}^{*}\left(\overline{\hat{\mu}}_{\hat{p}-1}^{*}, \overline{\hat{\mu}}_{\hat{p}}^{*}\right) \cdot \hat{T}_{\hat{p}-1}^{*}\left(\overline{\hat{\mu}}_{\hat{p}-2}^{*}, \overline{\hat{\mu}}_{\hat{p}-1}^{*}\right) \cdot \ldots \cdot \hat{T}_{1}^{*}\left(0, \overline{\hat{\mu}}_{1}^{*}\right) .
$$

Note that the decomposition (2.20) of the composite $\hat{T}^{*}\left(0, \mu^{*}\right)$ on $X$ is not unique, in general, and so it is not unique, in general, the decomposition (2.23). The decomposition 
(2.20) is unique if the testing $S$ converges to a finite subsequence of strict contractions so that $\mu^{*}=\sum_{i=1}^{p} \mu_{i}^{*}$ where $p$ is the maximum number of strict contractions of the decomposition (2.20) and the numbers $\mu_{i}^{*}, \forall i \in \bar{p}$ are unique. Uniqueness holds in the case that $p=\mu^{*}$ and $\mu_{i}^{*}=1, \forall i \in \bar{p}$.

Note that the assumption $\left(T_{\sigma(k n+j)}-T_{\sigma((k+1) n+j)}\right) \rightarrow 0$ uniformly in $X$ as $n \rightarrow \infty$ for some $k\left(\geq \mu^{*}\right) \in \mathbf{Z}_{0+}, \forall j \in \overline{k-1} \cup\{0\}$ in Theorem 2.5(iii) implies that $\left\{T_{\sigma(k n+j)}\right\} \rightarrow T_{j}^{*}=\hat{T}_{j}^{*}(0,1)$, $\forall j \in \overline{k-1} \cup\{0\}$. Note also that $k \geq \mu^{*}$ is a consequence of the fact that (2.20) is a decomposition of strict contraction while some of the limit self-mappings on $X$ reached as a result of the uniform convergence constraint $\left\{T_{\sigma(k n+j)}\right\} \rightarrow T_{j}^{*}=\hat{T}_{j}^{*}(0,1), \forall j \in \overline{k-1} \cup\{0\}$ can be expansive.

Theorem 2.6 Consider the iterative process (2.1) under the assumptions of Lemma 2.2. Then the following properties hold:

(i) If Lemma 2.2(ii) holds then $\left\{x_{n_{k}}\right\}$ and $\left\{x_{n_{k}}, x_{n_{k}+1}\right\}$, with $n_{k} \in S, \forall k \in \mathbf{Z}_{0_{+}}$, are Cauchy sequences, so bounded, and then convergent in $X$ if, in addition, $(X, d)$ is complete.

(ii) If Lemma 2.2(iii) holds with $\rho^{*} \leq \rho /\left(\sum_{i=0}^{\mu-1} M_{0}^{i}\right), \forall k \in \mathbf{Z}_{0+}$, for some real $\rho \in(0,1)$ then property (i) holds.

(iii) If both Lemma 2.2(ii) and Theorem 2.5(i) hold then $\left\{x_{n_{k}+\sum_{i=0}^{j} \mu_{i}^{*}}\right\}$ are Cauchy sequences, so bounded, and then convergent in $X$ if, in addition, $(X, d)$ is complete for all $j \in \bar{p} \cup\{0\}$ and $n_{k} \in S, \forall k \in \mathbf{Z}_{0+}$ and $\forall S \in \hat{S}$, where $\hat{S}=\hat{S}(\sigma)=\left\{S=\left\{n_{k}\right\}\right\}$.

(iv) Assume that $(X, d)$ is a compact metric space and that Lemma 2.2(ii) and Theorem 2.5(i) both hold. Then Fix $\hat{T}\left(0, \mu^{*}\right)=\{z\}$ for some $z \in X$ and $\hat{T}\left(0, \mu^{*}\right): X \rightarrow X$ is a strict Picard self-mapping and $\left\{x_{n_{k}+\bar{\mu}_{j}^{*}}\right\} \rightarrow z$ for any initial $x_{0} \in X$, where $\bar{\mu}_{j}^{*}=\sum_{i=1}^{j} \mu_{i}^{*}, \forall j \in \bar{p}$. If $q_{0 c}=\emptyset$ then the above result holds if $(X, d)$ is a complete metric space.

Proof If Lemma 2.2(i) holds then $\left\{x_{n_{k}}\right\}$ is a Cauchy sequence from (2.16), then bounded and, in addition, convergent in $X$ if $(X, d)$ is complete. The properties are extendable to $\left\{x_{n_{k}}, x_{n_{k}+1}\right\}$ from (2.11). Thus, property (ii) has been proven. Property (ii) follows from property (i) since $\rho_{k} \rightarrow \rho^{*} \in(0,1)$ as $k \rightarrow \infty$ and $\rho^{*} \leq \rho /\left(\sum_{i=0}^{\mu-1} M_{0}^{i}\right), \forall k \in \mathbf{Z}_{0+}$, for some real $\rho, \rho^{*} \in(0,1)$ then for any given $\varepsilon \in \mathbf{R}_{+}$there are $\bar{k}=\bar{k}(\varepsilon) \in \mathbf{Z}_{0+}$ and $\bar{k}^{*}=\bar{k}^{*}(\varepsilon)(\geq \bar{k}) \in$ $\mathbf{Z}_{0+}$ such that for $k(>\bar{k}) \in \mathbf{Z}_{0+} \rho_{k} \leq \rho /\left(\sum_{i=0}^{\mu-1} M_{0}^{i}\right), \forall k \geq \bar{k}$ and $\rho_{k} \leq \rho^{*} /\left(\sum_{i=0}^{\mu-1} M_{0}^{i}\right), \forall k \geq \bar{k}^{*}$. To prove property (iii), note that if, in addition, Theorem $2.5(\mathrm{i})$ holds then $\left\{x_{n_{k+1}+\sum_{i=0}^{j} \mu_{i}^{*}}-\right.$ $\left.x_{n_{k}+\sum_{i=0}^{j} \mu_{i}^{*}}\right\} \rightarrow 0$ since $\hat{T}^{*}\left(0, \mu^{*}\right)=\hat{T}^{*}\left(0, \bar{\mu}^{*}\right)=\hat{T}_{p}^{*}\left(\bar{\mu}_{p-1}^{*}, \bar{\mu}_{p}^{*}\right) \cdot \hat{T}_{p-1}^{*}\left(\bar{\mu}_{p-2}^{*}, \bar{\mu}_{p-1}^{*}\right) \cdot \ldots \cdot \hat{T}_{1}^{*}\left(0, \bar{\mu}_{1}^{*}\right)$ are strict contractions from (2.20), $\forall j \in \bar{p}$ and $\left\{x_{n_{k}}\right\}$ is a Cauchy sequence from property (i) since by defining $\bar{\mu}_{j}^{*}=\sum_{i=1}^{j} \mu_{i}^{*}, \bar{\rho}_{j}^{*}=K\left(0, \bar{\mu}_{j}^{*}\right)<1, \forall j \in \bar{p}$, one gets

$$
\begin{aligned}
d\left(x_{n_{k+1}+\bar{\mu}_{j}^{*}}, x_{n_{k}+\bar{\mu}_{j}^{*}}\right) & \leq d\left(x_{n_{k}}, x_{n_{k}+\bar{\mu}_{j}^{*}}\right)+d\left(x_{n_{k}}, x_{n_{k+1}}\right)+d\left(x_{n_{k+1}}, x_{n_{k+1}+\bar{\mu}_{j}^{*}}\right) \\
& \leq d\left(x_{n_{k}}, x_{n_{k+1}}\right)+\frac{2}{1-\bar{\rho}_{j}^{*}} \max \left(d\left(x_{n_{k}}, x_{n_{k}+1}\right), d\left(x_{n_{k+1}}, x_{n_{k+1}+1}\right)\right),
\end{aligned}
$$

$\forall j \in \bar{p}, \forall k \in \mathbf{Z}_{0_{+}}$, and, from property (i), one gets

$$
\lim _{k \rightarrow \infty} d\left(x_{n_{k+1}+\bar{\mu}_{j}^{*}}, x_{n_{k}+\bar{\mu}_{j}^{*}}\right)=\lim _{k \rightarrow \infty} d\left(x_{n_{k+1}+\bar{\mu}_{j}^{*}}, x_{n_{k}+\bar{\mu}_{j}^{*}}\right),
$$




$$
\begin{aligned}
& \lim _{k \rightarrow \infty} d\left(\hat{T}\left(n_{k+1}+\bar{\mu}_{j}^{*}, n_{k}+\bar{\mu}_{j}^{*}\right) x_{n_{k}+\bar{\mu}_{j}^{*}}, x_{n_{k}+\bar{\mu}_{j}^{*}}\right) \\
& =d\left(\lim _{k \rightarrow \infty}\left(\hat{T}\left(n_{k+1}+\bar{\mu}_{j}^{*}, n_{k}+\bar{\mu}_{j}^{*}\right) x_{n_{k}+\bar{\mu}_{j}^{*}}\right), \lim _{k \rightarrow \infty} x_{n_{k}+\bar{\mu}_{j}^{*}}\right) \\
& =d\left(\lim _{k \rightarrow \infty}\left(\hat{T}\left(n_{k+1}+\bar{\mu}_{j}^{*}, n_{k}+\bar{\mu}_{j}^{*}\right) x_{n_{k}+\bar{\mu}_{j}^{*}}\right), \lim _{k \rightarrow \infty}\left(\hat{T}\left(0, \bar{\mu}_{j}^{*}\right) x_{n_{k}}\right)\right) \\
& =d\left(\lim _{k \rightarrow \infty}\left(\hat{T}\left(\bar{\mu}_{j}^{*}, \bar{\mu}_{j}^{*}+\mu^{*}\right) \hat{T}\left(0, \bar{\mu}_{j}^{*}\right) x_{n_{k}}\right), \lim _{k \rightarrow \infty}\left(\hat{T}\left(0, \bar{\mu}_{j}^{*}\right) x_{n_{k}}\right)\right) \\
& =d\left(\hat{T}\left(\bar{\mu}_{j}^{*}, \bar{\mu}_{j}^{*}+\mu^{*}\right) \hat{T}\left(0, \bar{\mu}_{j}^{*}\right) \lim _{k \rightarrow \infty} x_{n_{k}}, \hat{T}\left(0, \bar{\mu}_{j}^{*}\right) \lim _{k \rightarrow \infty} x_{n_{k}}\right) \\
& =d\left(\hat{T}\left(\bar{\mu}_{j}^{*}, \bar{\mu}_{j}^{*}+\mu^{*}\right) \hat{T}\left(0, \bar{\mu}_{j}^{*}\right) z, \hat{T}\left(0, \bar{\mu}_{j}^{*}\right) z\right)=0, \quad \forall j \in \bar{p}
\end{aligned}
$$

since $\left\{x_{n_{k}}\right\} \rightarrow z$ from property (i) and the interchange of the limit and the distance function from the Lipschitz-continuity of the contraction self-mappings $\hat{T}\left(0, \bar{\mu}_{j}^{*}\right)$ and $\hat{T}\left(\bar{\mu}_{j}^{*}, \bar{\mu}_{j}^{*}+\right.$ $\left.\mu^{*}\right) \hat{T}\left(0, \bar{\mu}_{j}^{*}\right)$ on $\forall j \in \bar{p}$. Then $\left\{x_{n_{k}+\bar{\mu}_{j}^{*}}\right\} \rightarrow z_{j}=\hat{T}\left(0, \bar{\mu}_{j}^{*}\right) z$ and $\left\{x_{n_{k+1}+\bar{\mu}_{j}^{*}}\right\} \rightarrow z_{j}=\hat{T}\left(\bar{\mu}_{j}^{*}, \bar{\mu}_{j}^{*}+\right.$ $\left.\mu^{*}\right) \hat{T}\left(0, \bar{\mu}_{j}^{*}\right) z$ are Cauchy sequences, $\forall j \in \bar{p}$, and then bounded. If, in addition, $(X, d)$ is complete then $z_{j} \in X, \forall j \in \bar{p}$. Property (iii) has been proven for a sequence $S=\left\{n_{k}\right\}$. The proof for $\hat{S}=\left\{\left\{n_{k}\right\}\right\}$ is similar. On the other hand, one gets from (2.25)

$$
d\left(\hat{T}\left(0, \mu^{*}\right) z_{j}, z_{j}\right)=0, \quad \forall j \in \bar{p}
$$

then $\hat{T}\left(0, \mu^{*}\right) z_{j}=z_{j}$ so that $z_{j} \in$ Fix $\hat{T}\left(0, \mu^{*}\right), \forall j \in \bar{p}$. Since $\hat{T}\left(0, \mu^{*}\right): X \rightarrow X$ is a strict contraction from Lemma 2.2(ii), it has a unique fixed point from Banach contraction principle since $(X, d)$ is a compact metric space (i.e. totally bounded and complete, equivalently, if it very family of closed subsets of $X$ with finite intersection property has a nonempty intersection). Then $z_{j}=z \in$ Fix $\hat{T}\left(0, \mu^{*}\right)=\{z\}, \forall j \in \bar{p}$ and $\hat{T}\left(0, \mu^{*}\right) z=z$ for some $z \in X$. As a result, $\left\{x_{n_{k}+\bar{\mu}_{j}^{*}}\right\} \rightarrow z$ for any initial condition $x_{0} \in X, \forall j \in \bar{p}$. If there is no contractive selfmapping on $X$ not being a strict contraction in the switching law, the above holds if $(X, d)$ is just a complete metric space.

Note that Theorem 2.6(iv) holds even if $\hat{T}_{i}^{*}\left(\bar{\mu}_{i-1}^{*}, \bar{\mu}_{i}^{*}\right): X \rightarrow X$ for some $i \in \bar{p}$ is not contractive. However, the composite self-mapping $\hat{T}\left(0, \mu^{*}\right): X \rightarrow X$ is a strict Picard selfmapping and $\left\{x_{n_{k}+\mu_{j}^{*}}\right\} \rightarrow z, \forall j \in \bar{p}$, [1]. The error estimates and convergence rate are characterized in the subsequent result.

Theorem 2.7 Assume that $(X, d)$ is a compact (complete if $q_{0 c}=\emptyset$ ) metric space and that Lemma 2.2(ii) and Theorem 2.5(i) jointly hold. Then the following respective a priori and a posteriori error estimates and convergence rate hold for the iterative process (2.1) for any $x_{0} \in X$ if Fix $\hat{T}\left(0, \mu^{*}\right)=\{z\}$ :

$$
\begin{aligned}
& d\left(x_{n}, z\right) \leq\left(\frac{K \rho^{*^{n}}}{1-\rho^{*}}+o\left(\left|\varepsilon_{n}\right|\right)\right) d\left(x_{0}, x_{1}\right), \\
& d\left(x_{n}, z\right) \leq\left(\frac{K \rho^{*}}{1-\rho^{*}}+o\left(\left|\varepsilon_{n}\right|\right)\right) d\left(x_{n-1}, x_{n}\right), \\
& d\left(x_{n}, z\right) \leq\left(K \rho^{*^{n}}+o\left(\left|\varepsilon_{n}\right|\right)\right) d\left(x_{0}, z\right),
\end{aligned}
$$


$\forall n \geq n_{0}$ for any given $\varepsilon \in\left(0,1-\rho^{*}\right) \cap \mathbf{R}_{+}$, some real convergent sequence $\left\{\varepsilon_{n}\right\} \rightarrow 0$, with $\left|\varepsilon_{n}\right| \leq \varepsilon, \forall n \geq n_{0}$, and some $n_{0}=n_{0}(\varepsilon) \in \mathbf{Z}_{+}$and $K(\geq 1)=K(\varepsilon) \in \mathbf{R}_{+}$, where $\rho_{k} \leq$ $\rho /\left(\sum_{i=0}^{\mu} M_{0}^{i}\right)$ converges $\left\{\rho_{k}\right\} \rightarrow \rho^{*} \in(0,1)$.

Proof Given any $\varepsilon \in\left(0,1-\rho^{*}\right) \cap \mathbf{R}_{+}$, and since $\left\{\rho_{n_{k}}\right\} \rightarrow \rho^{*}$ from Lemma 2.2(ii), there are $n_{0 i}=n_{0 i}(\varepsilon) \in \mathbf{Z}_{+}(i=1,2,3)$ and $K_{a}(\geq 1)=K_{a}(\varepsilon) \in \mathbf{R}_{+}$and $K_{b}(\geq 1)=K_{b}(\varepsilon) \in \mathbf{R}_{+}$such that $\rho_{n_{k}}=\rho^{*}+\varepsilon_{n_{k}} \leq \rho^{*}+\varepsilon$ with $\left|\varepsilon_{n_{k}}\right| \leq \varepsilon, \forall n_{k} \geq n_{01}$ (since $\left\{\varepsilon_{n_{k}}\right\} \rightarrow 0$ ) and

$$
d\left(x_{n_{02}}, x_{n_{02}+1}\right) \leq K_{a} \rho^{*^{n} 02} d\left(x_{0}, x_{1}\right), \quad d\left(x_{n_{03}}, z\right) \leq K_{b} \rho^{*^{n} 03} d\left(x_{0}, z\right),
$$

where $z \in X$ is the unique element in the set Fix $\hat{T}\left(0, \mu^{*}\right)$, since $n_{02}$ and $n_{03}$ are finite and $\min \left(K_{a}, K_{b}\right) \geq 1$, one gets for the testing sequence $S=S(\sigma)=\left\{n_{k}\right\}$ of a solution of the iterative scheme (2.1)

$$
\begin{aligned}
& \max \left(d\left(x_{n_{02}}, x_{n_{02}+1}\right), d\left(x_{n_{k}-1}, x_{n_{k}}\right), d\left(x_{n_{k}-1}, z\right)\right) \\
& \quad \leq \max \left(K_{a}, K_{b}\right) \max \left(\rho^{*^{n_{02}}} d\left(x_{0}, x_{1}\right), d\left(x_{n_{k}-1}, x_{n_{k}}\right), \rho^{*^{n_{03}}} d\left(x_{n_{k}-1}, z\right)\right),
\end{aligned}
$$

$\forall n \geq n_{02}$ since $\left\{x_{n_{k}}\right\} \rightarrow z(\in X)$ from Theorem 2.5(i) and Theorem 2.6(iv) which holds since Lemma 2.2(ii) and Theorem 2.5(i) hold and $(X, d)$ is a compact (it suffices it be complete if $\left.q_{0 c}=\emptyset\right)$ metric space. Then one gets by taking $K^{\prime}=\max \left(K_{a}, K_{b}\right)$ and $n_{0}=$ $\max \left(n_{01}, n_{02}, n_{03}\right)$ :

$$
\begin{aligned}
& d\left(x_{n_{k}}, z\right) \leq\left(\frac{\rho^{*^{n_{k}-n_{02}}}+o\left(\left|\varepsilon_{n_{k}}\right|\right)}{1-\rho^{*}-\varepsilon}\right) d\left(x_{n_{02}}, x_{n_{1}}\right) \leq K_{a}\left(\frac{\rho^{*^{n_{k}}}+\rho^{*^{n_{02}}} o\left(\left|\varepsilon_{n_{k}}\right|\right)}{1-\rho^{*}-\varepsilon}\right) d\left(x_{0}, x_{1}\right) \\
& \leq\left(\frac{K_{a} \rho^{*^{n_{k}}}}{1-\rho^{*}-\varepsilon}+o\left(\left|\varepsilon_{n_{k}}\right|\right)\right) d\left(x_{0}, x_{1}\right) \leq\left(\frac{K^{\prime} \rho^{*^{n_{k}}}}{1-\rho^{*}}+o\left(\left|\varepsilon_{n_{k}}\right|\right)\right) d\left(x_{0}, x_{1}\right) \\
& d\left(x_{n_{k}}, z\right) \leq\left(\frac{K^{\prime} \rho^{*^{n_{k}}}}{1-\rho^{*}}+o\left(\left|\varepsilon_{n_{k}}\right|\right)\right) d\left(x_{0}, z\right) \leq\left(\frac{\rho^{*}}{1-\rho^{*}-\varepsilon}+o\left(\left|\varepsilon_{n_{k}}\right|\right)\right) d\left(x_{n_{k}-1}, x_{n_{k}}\right) \\
& \leq\left(\frac{K^{\prime} \rho^{*}}{1-\rho^{*}}+o\left(\left|\varepsilon_{n_{k}}\right|\right)\right) d\left(x_{n_{k}-1}, x_{n_{k}}\right) \text {, }
\end{aligned}
$$

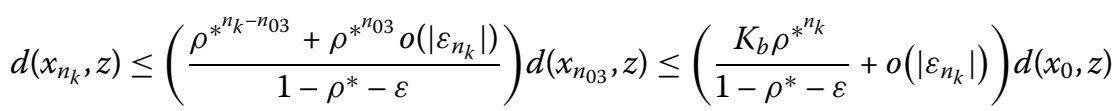

$$
\begin{aligned}
& \leq\left(\frac{K^{\prime} \rho^{*^{n_{k}}}}{1-\rho^{*}}+o\left(\left|\varepsilon_{n_{k}}\right|\right)\right) d\left(x_{0}, z\right),
\end{aligned}
$$

since $\frac{1}{1-\rho^{*}-\varepsilon}<\frac{1}{1-\rho^{*}}, \frac{K_{a} \rho^{*^{n} 02} o\left(\left|\varepsilon_{n_{k}}\right|\right)}{1-\rho^{*}-\varepsilon}=o\left(\left|\varepsilon_{n_{k}}\right|\right)$ and $\frac{K_{b} \rho^{*^{n} 03} o\left(\left|\varepsilon_{n_{k}}\right|\right)}{1-\rho^{*}-\varepsilon}=o\left(\left|\varepsilon_{n_{k}}\right|\right)$. Now, denote in (2.20) the Lipschitz constants of the limit self-mappings on $X$ which define the composite limit self-mapping $\hat{T}^{*}\left(0, \mu^{*}\right)$ on $X$ by $K_{j}^{*}=K_{j}\left(0, \mu_{j}^{*}\right), \forall j \in \bar{p}$. Now, note from (2.32) and the triangle inequality that the sequence $\left\{x_{n}\right\}$, which contains the testing subsequence $\left\{x_{n_{k}}\right\}$, satisfies for any $j \in \overline{n_{k+1}-n_{k}-1}, \forall k\left(\geq n_{0}\right) \in \mathbf{Z}_{+}$:

$$
\begin{aligned}
d\left(x_{n_{k}+j}, z\right) & \leq d\left(x_{n_{k}}, z\right)+\sum_{i=1}^{j} d\left(x_{n_{k}+i}, x_{n_{k}+i-1}\right) \\
& \leq\left(1+K_{1}\left(n_{k}, n_{k}+1\right)+K_{1}\left(n_{k}, n_{k}+1\right) K_{2}\left(n_{k}+1, n_{k}+2\right)+\cdots\right.
\end{aligned}
$$




$$
\begin{aligned}
& \left.+K_{1}\left(n_{k}, n_{k}+1\right) K_{2}\left(n_{k}+1, n_{k}+2\right) \cdots K_{j}\left(n_{k}+j-1, n_{k}+j\right)\right) d\left(x_{n_{k}}, z\right) \\
& \leq\left[\left(1+K_{1}^{*}+K_{1}^{*} K_{2}^{*}+\cdots+K_{1}^{*} K_{2}^{*} K_{3}^{*} \cdots K_{j}^{*}\right)+\left(K_{1}\left(n_{k}, n_{k}+1\right)-K_{1}^{*}\right)\right. \\
& +\left(K_{1}\left(n_{k}, n_{k}+1\right) K_{2}\left(n_{k}+1, n_{k}+2\right)-K_{1}^{*} K_{2}^{*}\right)+\cdots \\
& +\left(K_{1}\left(n_{k}, n_{k}+1\right) K_{2}\left(n_{k}+1, n_{k}+2\right) \cdots K_{j}\left(n_{k}+j-1, n_{k}+j\right)\right. \\
& \left.\left.-K_{1}^{*} K_{2}^{*} K_{3}^{*} \cdots K_{j}^{*}\right)\right] \\
& \times \rho^{*^{\left(n_{k}+j-n\right)}}\left[\left(\frac{K^{\prime} \rho^{*^{n}}}{1-\rho^{*}}+o\left(\varepsilon_{n}\right)\right)+\left|o\left(\left|\varepsilon_{n_{k}}\right|\right)-o\left(\left|\varepsilon_{n}\right|\right)\right|\right] d\left(x_{0}, x_{1}\right) \\
& \leq\left[1+p\left(\max _{1 \leq j \leq p}\left(K_{j}^{*}, K_{j}^{* p}\right)+\max _{1 \leq j \leq p}\left(K\left(n_{k}, n_{k}+j\right)-\prod_{i=1}^{j}\left[K_{i}^{*}\right]\right)\right)+\cdots\right. \\
& +\left(K_{1}\left(n_{k}, n_{k}+1\right) K_{2}\left(n_{k}+1, n_{k}+2\right) \cdots K_{j}\left(n_{k}+j-1, n_{k}+j\right)\right. \\
& \left.\left.-K_{1}^{*} K_{2}^{*} K_{3}^{*} \cdots K_{j}^{*}\right)\right] \rho^{*^{\left(n_{k}+j-n\right)}} \\
& \times\left[\left(\frac{K^{\prime} \rho^{*^{n}}}{1-\rho^{*}}+o\left(\left|\varepsilon_{n}\right|\right)\right)+\left|o\left(\left|\varepsilon_{n_{k}}\right|\right)-o\left(\left|\varepsilon_{n}\right|\right)\right|\right] d\left(x_{0}, x_{1}\right) \\
& \leq M\left(n_{k}\right)\left[\left(\frac{K^{\prime} \rho^{*^{n}}}{1-\rho^{*}}+o\left(\left|\varepsilon_{n}\right|\right)\right)\right] d\left(x_{0}, x_{1}\right) \\
& +M\left(n_{k}\right)\left|o\left(\left|\varepsilon_{n_{k}}\right|\right)-o\left(\left|\varepsilon_{n}\right|\right)\right| d\left(x_{0}, x_{1}\right)
\end{aligned}
$$

for any $j \in \overline{n_{k+1}-n_{k}-1}$, where $\varepsilon_{j}=\rho_{j}-\rho^{*}$ with $\rho^{*} \in(0,1), \forall j \in \mathbf{Z}_{0+}$, and

$$
\begin{aligned}
M\left(n_{k}\right)= & \max _{1 \leq j \leq p}\left(\left[1+p\left(\max _{1 \leq j \leq p}\left(K_{j}^{*}, K_{j}^{* p}\right)+\max _{1 \leq j \leq p}\left(K\left(n_{k}, n_{k}+j\right)-\prod_{i=1}^{j}\left[K_{i}^{*}\right]\right)\right)+\cdots\right.\right. \\
& +\left(K_{1}\left(n_{k}, n_{k}+1\right) K_{2}\left(n_{k}+1, n_{k}+2\right) \cdots K_{j}\left(n_{k}+j-1, n_{k}+j\right)\right. \\
& \left.\left.\left.-K_{1}^{*} K_{2}^{*} K_{3}^{*} \cdots K_{j}^{*}\right)\right]\right)<+\infty
\end{aligned}
$$

and one gets from (2.35) for any integer $n=n_{k}+j, \forall j \in \overline{n_{k+1}-n_{k}-1}, \forall k \in \mathbf{Z}_{0+}$ with $K=$ $K^{\prime} \sup _{k \geq n_{0}} M\left(n_{k}\right)=\max \left(K_{a}, K_{b}\right) \sup _{k \geq n_{0}} M\left(n_{k}\right)$ that the $a$ priori error estimate satisfies

$$
d\left(x_{n}, z\right) \leq\left[\left(\frac{K^{\prime} M\left(n_{k}\right) \rho^{*^{n}}}{1-\rho^{*}}\right)+o\left(\left|\varepsilon_{n}\right|+\left|\varepsilon_{n_{k}}\right|\right)\right] d\left(x_{0}, x_{1}\right), \quad \forall n \in\left[n_{k}, n_{k+1}\right),
$$

$\forall k \in \mathbf{Z}_{0+}$

and, since $\left\{\varepsilon_{n_{k}}:\left(n_{k} \geq n_{0} \wedge k \in \mathbf{Z}_{0+}\right)\right\}$ and $\left\{\varepsilon_{n}: n \geq n_{0}\right\}$ subject to $\left\{\varepsilon_{n_{k}}:\left(n_{k} \geq n_{0} \wedge k \in\right.\right.$ $\left.\left.\mathbf{Z}_{0+}\right)\right\} \subseteq\left\{\varepsilon_{n}: n \geq n_{0}\right\}$, with both sequences converging to zero,

$$
d\left(x_{n}, z\right) \leq\left[\left(\frac{K \rho^{*^{n}}}{1-\rho^{*}}\right)+o\left(\left|\varepsilon_{n}\right|\right)\right] d\left(x_{0}, x_{1}\right), \quad \forall n \in\left[n_{k}, n_{k+1}\right), \forall k \in \mathbf{Z}_{0+}
$$

and $\left(d\left(x_{n}, z\right) / d\left(x_{0}, x_{1}\right)\right) \rightarrow 0$ as $n \rightarrow \infty$ at exponential rate so that (2.27) is proven. Closely analogous proofs to that of (2.27) follow directly for (2.28) and (2.29). 
A discussion of cases of interest concerning the above result follows.

Remark 2.8 (1) Theorem 2.7 refers to the case when $\left\{\hat{T}\left(n_{k}, n_{k+1}\right)\right\} \rightarrow \hat{T}^{*}\left(0, \mu^{*}\right)$ with $\hat{T}^{*}\left(0, \mu^{*}\right)$ being a composite strictly contractive self-mapping on $X$ of the form (2.20), i.e. possessing $p$ (non-necessarily strictly contractive) fixed configurations which are the limit of the switching law $\sigma: \mathbf{Z}_{0+} \rightarrow \bar{q} \subseteq \mathbf{Z}_{+} . \hat{T}^{*}\left(0, \mu^{*}\right)$ is a strict Picard self-mapping on $X$ as a result. Also, $\left\{\hat{T}(n+1,0) x_{0}\right\} \rightarrow z$, satisfying (2.27)-(2.29), and $\left\{\hat{T}^{*^{n}}\left(0, \mu^{*}\right) x_{0}\right\} \rightarrow z$, satisfying (2.27)-(2.29) with $K=1$ and $o\left(\left|\varepsilon_{n}\right|\right)$ being replaced with 0 , for any given $x_{0} \in X$, where Fix $\hat{T}^{*}\left(0, \mu^{*}\right)=\{z\}$.

(2) A particular case of interest of Theorem 2.7 is that when $\hat{T}^{*}\left(0, \mu^{*}\right)=\hat{T}^{*}(0,1)=$ $\hat{T}_{j}^{*}(0,1)=T_{j}^{*}$, so that $\left\{T_{\sigma(n)}\right\} \rightarrow T_{j}^{*}$, with $\mu^{*}=1$ and some $j \in \bar{q}$ (one of the configurations of the switching law) so that the limit self-mapping $T_{j}^{*}$ on $X$ is a strict contraction, and then a strict Picard self-mapping, and the switching law $\sigma: \mathbf{Z}_{0+} \rightarrow \bar{q} \subseteq \mathbf{Z}_{+}$is such that $\left\{T_{\sigma(n)}\right\} \rightarrow T_{j}^{*}$ uniformly in $X$. Since the testing switching $\left\{n_{k}\right\}$ has the property $\lim _{k \rightarrow \infty}\left(n_{k+1}-n_{k}\right)=1, \lim _{k \rightarrow \infty} M\left(n_{k}\right)=\lim _{n \rightarrow \infty} M(n)=1$ so that an admissible choice of $K=\max \left(K_{a}, K_{b}\right)$ can be made in (2.27)-(2.29). The interpretation of the presence of the real constant $K \neq 1$ in the error estimates and convergence rate is due to the fact that the sequence of composite self-mappings $\{\hat{T}(0, n)\}$ governed by the switching law to build the iterative scheme $x_{n+1}=T_{n} x_{n}=\hat{T}(n+1,0) x_{0}$ for any given $x_{0} \in X$ is not of the form $\left\{T_{j}^{*^{n}}\right\}$, while $\left\{T_{\sigma(n)}\right\}$ converges to a uniform limit strictly contractive $T_{j}^{*}$ on $X$ with a unique fixed point $z_{j} \in X$ subject to $\lim _{k \rightarrow \infty}\left(n_{k+1}-n_{k}\right)=1$ and $\left\{\hat{T}(n+1,0) x_{0}\right\} \rightarrow z_{j}$ and $\left\{T_{j}^{*^{n}} x_{0}\right\} \rightarrow z_{j}$ for any given $x_{0} \in X$ according to (2.27)-(2.29) with the replacement $z \rightarrow z_{j}$.

(3) Particular cases of interest of Remark 2.8(1)-(2) are when fixed points $z=0$, respectively, $z_{j}=0$ (which are also, in particular, globally asymptotically stable equilibrium points if the iterative scheme refers to a dynamic system). Thus, the iterative scheme converges exponentially fast to zero. If the formalism is concerned with a Banach space $(X,\|\cdot\|)$ endowed with the norm $\|\cdot\|$, with $X$ being a linear space, we can use as metric the norminduced metric so that (2.27)-(2.29) become

$$
\begin{aligned}
\left\|x_{n}\right\| & \leq\left(\frac{K \rho^{*^{n}}}{1-\rho^{*}}+o\left(\left|\varepsilon_{n}\right|\right)\right)\left\|x_{1}-x_{0}\right\| \leq\left(\frac{K\left\|T_{\sigma(0)}-1\right\| \rho^{*^{n}}}{1-\rho^{*}}+o\left(\left|\varepsilon_{n}\right|\right)\right)\left\|x_{0}\right\|, \\
\left\|x_{n}\right\| & \leq\left(\frac{K \rho^{*}}{1-\rho^{*}}+o\left(\left|\varepsilon_{n}\right|\right)\right)\left\|x_{n}-x_{n-1}\right\| \\
& \leq\left(\frac{K\|\hat{T}(n, 0)-\hat{T}(n-1,0)\| \rho^{*}}{1-\rho^{*}}+o\left(\left|\varepsilon_{n}\right|\right)\right)\left\|x_{0}\right\|, \\
\left\|x_{n}\right\| & \leq\left(K \rho^{*^{n}}+o\left(\left|\varepsilon_{n}\right|\right)\right)\left\|x_{0}\right\|,
\end{aligned}
$$

$\forall n \geq n_{0}$. If a fixed point of $z \neq 0$ then (2.39)-(2.41) hold in closed forms under the replacements $\left\|x_{n}\right\| \rightarrow\left\|x_{n}-z\right\|, \forall n \geq n_{0}$ and $\left\|x_{0}\right\| \rightarrow\left\|x_{0}-z\right\|$.

(4) Note that in the case that $\rho^{*}=1$, the limit self-mapping $\hat{T}\left(0, \mu^{*}\right)$ is only guaranteed to be non-expansive. Then (2.39) and (2.40) do not hold. However, one gets from Theorem 2.7 and (2.41), with the replacements $\left\|x_{n}\right\| \rightarrow\left\|x_{n}-z\right\|, \forall n \geq n_{0}$ and $\left\|x_{0}\right\| \rightarrow\left\|x_{0}-z\right\|$,

$$
\left\|x_{n}-z\right\| \leq\left(K+o\left(\left|\varepsilon_{n}\right|\right)\right)\left\|x_{0}-z\right\|, \quad \forall n \geq n_{0}
$$


provided that the non-expansive self-mapping $\hat{T}^{*}\left(0, \mu^{*}\right)$ on $X$ has a fixed point $z \in X$. Then one has $\left|\lim \sup _{n \rightarrow \infty}\left\|x_{n}\right\|-\|z\|\right| \leq \lim \sup _{n \rightarrow \infty}\left\|x_{n}-z\right\| \leq K\left\|x_{0}-z\right\|+\|z\|$ so that $\lim \sup _{n \rightarrow \infty}\left\|x_{n}\right\| \leq K\left\|x_{0}\right\|+(1+K)\|z\|$ and $\left\{x_{n}\right\}$ is bounded for any initial $x_{0} \in X$ although convergence to the fixed point is not guaranteed. This is a well-known result from fixed point theory for non-expansive self-mappings and a well-known (non-asymptotic) global stability result in related problems of stability of dynamic systems which can have a global attractor which can be either a fixed point, which is also an equilibrium point which is not asymptotically stable, or a stable limit cycle. If $z=0$ then the above result takes the simpler form $\lim \sup _{n \rightarrow \infty}\left\|x_{n}\right\| \leq K\left\|x_{0}\right\|$.

Remark 2.9 The given convergence properties to fixed points also hold if $\left\{\hat{T}\left(n_{k}, n_{k+1}\right)\right\} \rightarrow$ $\hat{T}\left(0, \mu^{*}\right)$ point-wisely in $X$ and $\hat{T}\left(0, \mu^{*}\right)$ is a strict contraction on $X$. Assume that $\left\{\hat{T}\left(n_{k}, n_{k+1}\right)\right\} \rightarrow \hat{T}\left(0, \mu^{*}\right)$ with $\left\{\hat{T}\left(n_{k}, n_{k+1}\right) x_{n_{k}}\right\} \rightarrow x$ and $\left\{\hat{T}\left(0, \mu^{*}\right) x_{0}\right\} \rightarrow z$ so that

$$
\begin{aligned}
d(x, z) \leq & d\left(x, \hat{T}\left(n_{k}, n_{k+1}\right) x_{n_{k}}\right)+d\left(\hat{T}\left(n_{k}, n_{k+1}\right) x_{n_{k}}, \hat{T}\left(0, \mu^{*}\right) x_{n_{k}}\right) \\
& +d\left(\hat{T}\left(0, \mu^{*}\right) x_{n_{k}}, z\right)+d\left(\hat{T}\left(0, \mu^{*}\right) z, z\right) \\
\leq & d\left(x, \hat{T}\left(n_{k}, n_{k+1}\right) x_{0}\right)+\left(\hat{T}\left(n_{k}, n_{k+1}\right) x_{n_{k}}, \hat{T}\left(n_{k}, n_{k+1}\right) x_{0}\right) \\
& +d\left(\hat{T}\left(n_{k}, n_{k+1}\right) x_{n_{k}}, \hat{T}\left(0, \mu^{*}\right) x_{n_{k}}\right) \\
& +d\left(\hat{T}\left(0, \mu^{*}\right) x_{n_{k}}, z\right)+d\left(\hat{T}\left(0, \mu^{*}\right) z, z\right), \quad \forall k \in \mathbf{Z}_{0+} .
\end{aligned}
$$

Then $\left\{d\left(\hat{T}\left(n_{k}, n_{k+1}\right) x_{n_{k}}, \hat{T}\left(0, \mu^{*}\right) x_{n_{k}}\right)\right\} \rightarrow 0$ from point-wise convergence, $\left\{d\left(\hat{T}\left(0, \mu^{*}\right) x_{n_{k}}\right.\right.$, $z)\} \rightarrow 0$, since $z$ is a fixed point of $\hat{T}\left(0, \mu^{*}\right),\left\{d\left(x, \hat{T}\left(n_{k}, n_{k+1}\right) x_{0}\right)\right\} \rightarrow x$ and $\left\{d\left(\hat{T}\left(n_{k}, n_{k+1}\right) x_{n_{k}}\right.\right.$, $\left.\left.\hat{T}\left(n_{k}, n_{k+1}\right) x_{0}\right)\right\} \rightarrow x$. Taking the limit as $k \rightarrow \infty$ in (2.43) yields $x=z$.

Example 2.10 Retaking the simple Example 2.1, the following conclusions arise. Assume that $\left\{T_{\sigma(n)}\right\} \rightarrow \hat{T}\left(0, \mu^{*}\right)=\left(T_{1} T_{3} T_{2}\right)^{\omega} T_{2}^{g}$ which is a composite self-mapping composed of three, in general, composite self-mappings, subject to nonzero integers $\omega>1$ and $g>\omega-1$ with $\alpha \beta<1$. In this case, there is a limit self-mapping $\hat{T}\left(0, \mu^{*}\right)$ which is a strict contraction and then a strict Picard mapping and has a unique fixed point $\{0\}$ to which any iteration built with (2.1) for any initial real condition converges. However, the decomposition in a maximum number of strict contractions is not unique since $0 \leq g_{1} \leq g$ can be chosen arbitrarily in the subsequent decomposition by taking into account that $T_{i}: X \rightarrow X, i=$ 1,2,3, commute:

$$
\begin{aligned}
\hat{T}\left(0, \mu^{*}\right) & =\left(T_{1} T_{3} T_{2}\right)^{\omega-1} \cdot\left(T_{1} T_{3} T_{2}^{g+1-\omega}\right)=\hat{T}_{1}\left(0, \mu_{1}^{*}\right) \cdot \hat{T}_{2}\left(\mu_{1}^{*}, \mu_{1}^{*}+\mu_{2}^{*}\right) \\
& =\left(T_{1} T_{3} T_{2}^{g+1-\omega}\right) \cdot\left(T_{1} T_{3} T_{2}\right)^{\omega-1}=\hat{T}_{1}\left(0, \mu_{01}^{*}\right) \cdot \hat{T}_{2}\left(\mu_{01}^{*}, \mu_{01}^{*}+\mu_{02}^{*}\right) \\
& =\left(T_{1} T_{3} T_{2}^{g}\right) \cdot\left(T_{1} T_{3}\right)^{\omega-1}=\hat{T}_{1}\left(0, \mu_{11}^{*}\right) \cdot \hat{T}_{2}\left(\mu_{11}^{*}, \mu_{11}^{*}+\mu_{12}^{*}\right)
\end{aligned}
$$

with $\omega^{*}=3 \omega+g, \mu_{2}^{*}=\omega^{*}-\mu_{1}^{*}$ and $\mu_{1}^{*}=3(\omega-1) \neq \mu_{01}^{*}=g+3-\omega<g+2$. But if $g=4 \omega-6$ then $\mu_{11}^{*}=g+2 \neq \mu_{01}^{*}<g+2($ since $\omega>1)$ if $g \neq 4 \omega-6$.

\section{Numerical examples}

This section contains some numerical examples regarding the theoretical results stated in the previous section. Two examples are discussed. The first one considers a scalar time- 


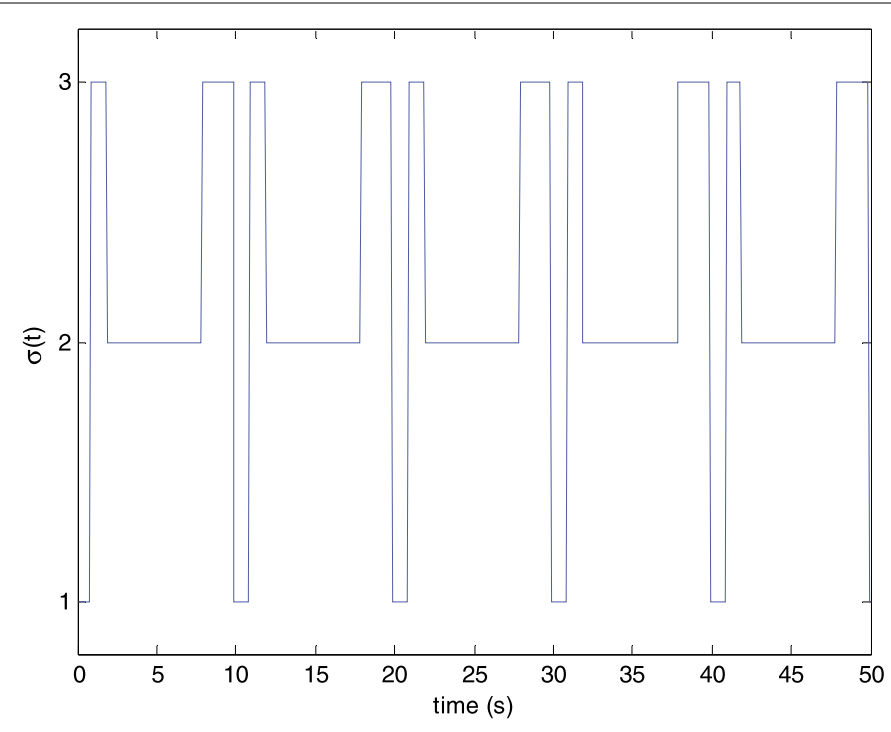

Figure 1 Switching law.

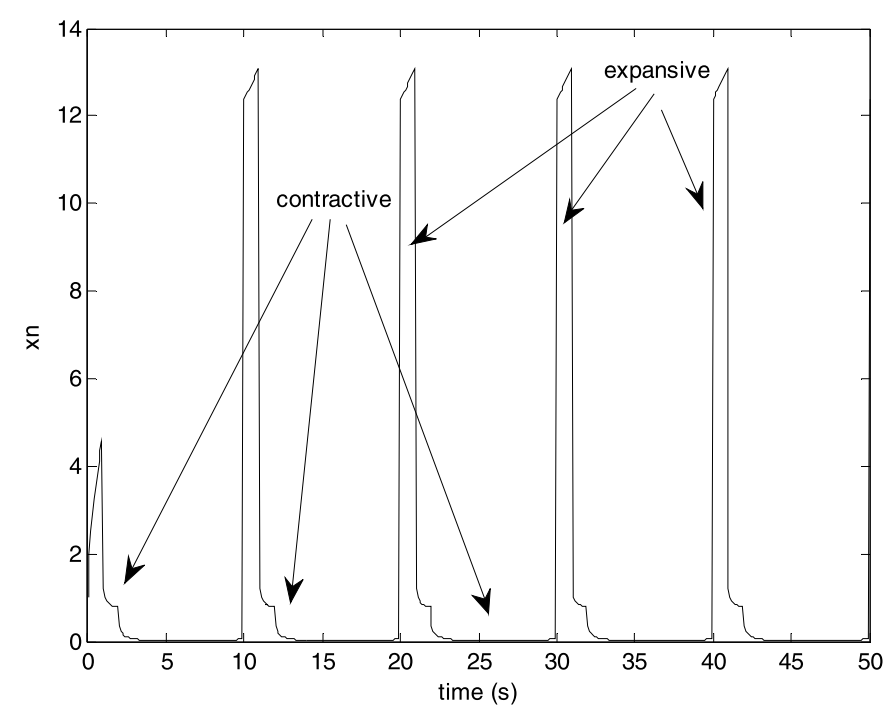

Figure 2 Solution trajectory for the switching rule depicted in Figure 1.

invariant nonlinear switched system while the second one deals with a linear time-varying switched system.

\subsection{Scalar nonlinear switched system}

Consider the nonlinear discrete-time dynamic system given by (2.1) with $\sigma(t) \in\{1,2,3\}$ and $T_{1}\left(x_{n}\right)=x_{n}+\frac{1}{x_{n}}, T_{2}\left(x_{n}\right)=0.95 x_{n} e^{-x_{n}}$ and $T_{3}\left(x_{n}\right)=1.2 \tanh \left(x_{n}\right)$. Note that $T_{1}\left(x_{n}\right)$ is a mixed type operator, being contractive for large $x_{n}$ while expansive for small values of $x_{n}$ (it is also non-strictly contractive in $[1, \infty)$ ), $T_{2}\left(x_{n}\right)$ is strictly contractive with $K_{2}=0.95$ and $T_{3}\left(x_{n}\right)$ is expansive with $K_{3}=1.2$. The stability of the nonlinear switched system (2.1) depends on the switching law. For instance, for the switching law depicted in Figure 1, the solution of the discrete-time system is shown in Figure 2. 


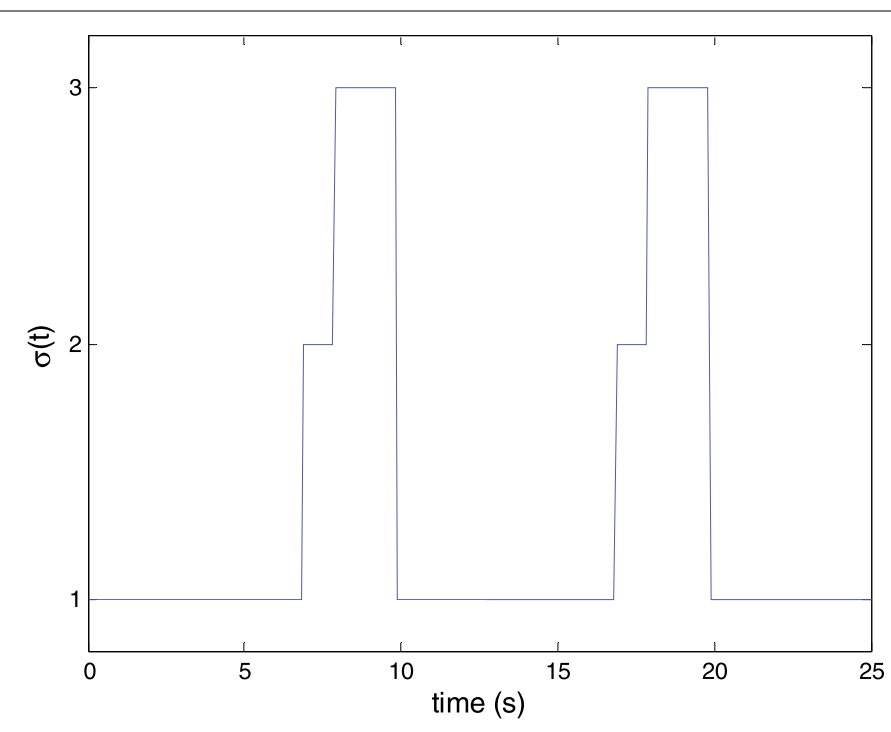

Figure 3 Switching rule.

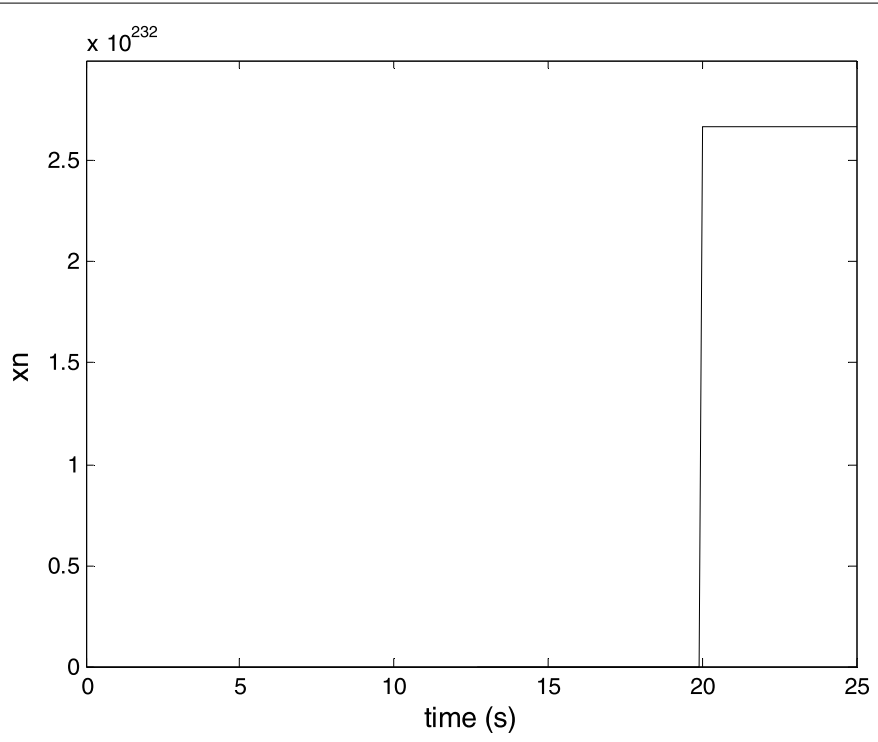

Figure 4 Solution trajectory for the switching rule depicted in Figure 3.

The contractive and expansive phases generated by the switching rule can be clearly appreciated in Figure 2. In this way, when the contractive operator, $T_{2}\left(x_{n}\right)$, is activated by the switching rule, the solution trajectory converges to zero while when the non-contractive operators are activated, the solution enlarges. Nevertheless, the combination of these operators according to the switching rule provides a globally stable system, with a bounded trajectory at all time. On the other hand, if the switching rule is given by Figure 3, the solution trajectory is displayed in Figure 4.

In this case, the trajectory enlarges very much as Figure 4 shows. The time scale in this figure has been reduced to handle the large values of the signal. Hence, a serious problem arises in this example: How can the stability of the switched system be proved under dif- 
ferent switching rules? The results stated in Section 2 can be used to solve this question as the following example shows.

\subsection{Linear time-varying switched dynamic system}

This example shows how the previously presented theoretical results can be applied to study the asymptotic stability of time-varying dynamical systems by means of their discretization. This problem is of great importance in practice since many control systems are currently designed in a discrete-time set-up which leads to iterative schemes of the form (2.1) despite the system being originally in continuous-time. Therefore, the results introduced above might be used in a highly practical context. For this purpose, a linear time-varying switched dynamical system will be considered. These types of systems are described by a number of different parameterizations which are themselves time-varying. The switching rule selects the active parameterization during each time interval, as discussed in Section 2. The stability of time-varying systems is far from being trivial and offers a variety of behaviors different from those of linear time-invariant ones. For instance, a linear time-varying system may possess constant stable eigenvalues (i.e. constant eigenvalues with negative real part) and, nevertheless, to be asymptotically unstable in the Lyapunov sense, [51]. The tools provided by fixed point theory are useful in this situation where other techniques become intricate. Therefore, consider a linear time-varying system of the form

$$
\dot{x}(t)=A_{\sigma(t)}(t) x(t)
$$

where $\sigma(t) \in\{1,2,3\}$ and the dynamics matrices are given by

$$
\begin{aligned}
& A_{\sigma(t)}(t)=\left(\begin{array}{cc}
\cos t & -\sin t \\
\sin t & \cos t
\end{array}\right), \quad B_{\sigma(t)}\left(\begin{array}{cc}
\cos t & \sin t \\
-\sin t & \cos t
\end{array}\right), \\
& B_{1}=\left(\begin{array}{cc}
-1 & -4 \\
0 & -1
\end{array}\right), \quad B_{2}=\left(\begin{array}{cc}
-1 & 4 \\
1 & -1
\end{array}\right), \quad B_{3}=\left(\begin{array}{ll}
0 & -1 \\
0 & -1
\end{array}\right) .
\end{aligned}
$$

It is easy to verify that matrices (3.2) are in the form $A_{\sigma(t)}=e^{\Omega t} B_{\sigma(t)} e^{-\Omega t}$, [52], with $\Omega=$ $\left(\begin{array}{ll}0 & -1 \\ 1 & 0\end{array}\right)$. In this way, the solution of (3.1) is given by

$$
x(t)=e^{\Omega t} e^{\left(-\Omega+B_{\sigma\left(t_{i}\right)}\right) t} x\left(t_{i}\right)
$$

[52], with $\sigma\left(t_{i}^{-}\right) \neq \sigma\left(t_{i}^{+}\right) \equiv \sigma\left(t_{i}\right)$ and $\sigma(t)=\sigma\left(t_{i}\right)$ for $t \in\left[t_{i}, t_{i+1}\right), \forall i \in \mathbf{Z}_{0+}$. The eigenvalues of $\Omega$ are $\pm j$ implying that the stability of (3.3) is directly influenced by that of the matrices $\left(-\Omega+B_{\sigma(t)}\right)$. Thus, we have

$$
\begin{aligned}
& \operatorname{spec}\left(-\Omega+B_{1}\right)=\{-1-j \sqrt{3},-1+j \sqrt{3}\}, \\
& \operatorname{spec}\left(-\Omega+B_{2}\right)=\{-1,-1\}, \\
& \operatorname{spec}\left(-\Omega+B_{3}\right)=\{-1,0\} .
\end{aligned}
$$

Therefore, the first parameterization leads to an unstable system, the second to a stable one while the last one is marginally stable. Under these circumstances, the stability of the switched system relies directly on the switching law. That is, there will be switching 


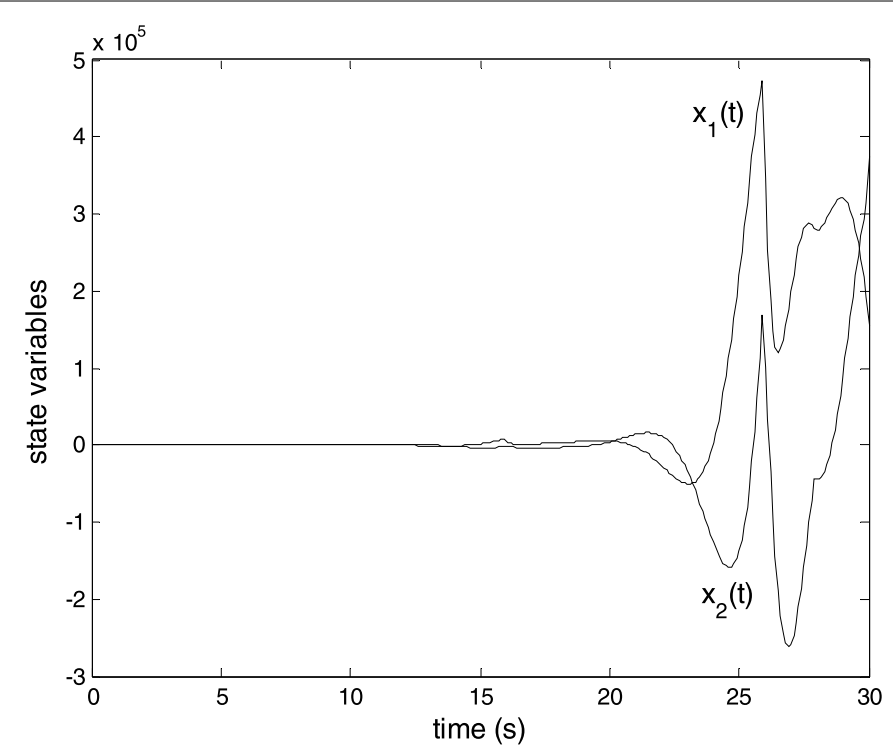

Figure 5 State variables evolution under switching rule (a).

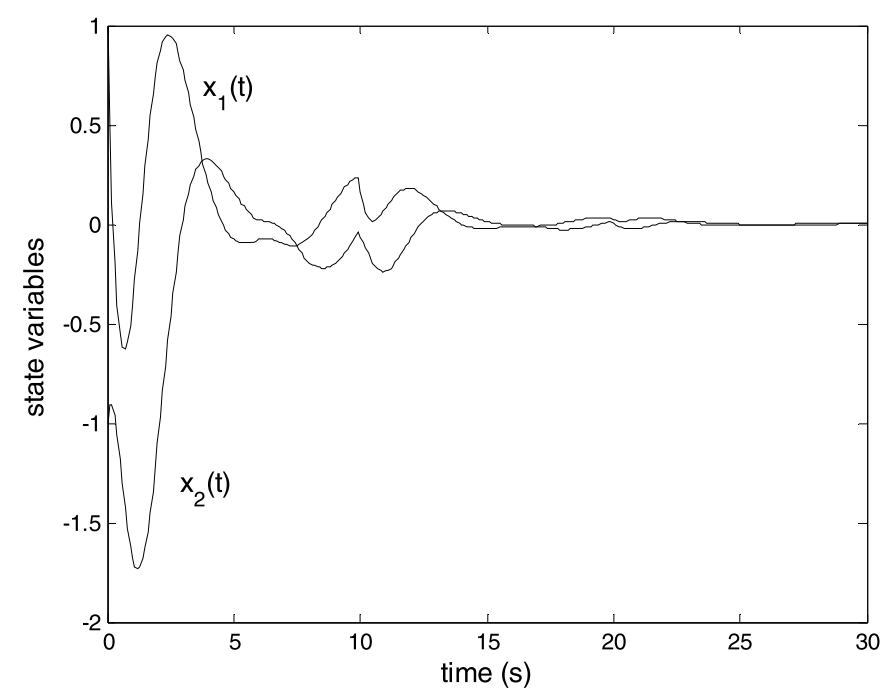

Figure 6 State variables evolution under switching rule (b).

laws under which the switched system is asymptotically stable while for others it may be unstable. To verify the stability of (3.1) under different switching laws, we will discretize the system so as to generate an iterative scheme of the form (2.1). Thus, from (3.3) if the sampling period is denoted by $h$ and $x(n h)=x_{n}$, we have after a little bit of algebra:

$$
x_{n+1}=e^{\Omega n h} e^{\Omega h} e^{\left(-\Omega+B_{\sigma(n h)}\right) h} e^{-\Omega n h} x_{n},
$$

which fits into the iterative structure (2.1) with operators $T_{\sigma(n)}=e^{\Omega n h} e^{\Omega h} e^{\left(-\Omega+B_{\sigma(n h)) h}\right.} e^{-\Omega n h}$. It is remarkable the complexity of these operators for the time-varying system (3.1)(3.2). Also, notice that all $e^{\Omega n h}, e^{\Omega h}$ and $e^{-\Omega n h}$ have eigenvalues with absolute value unity. 


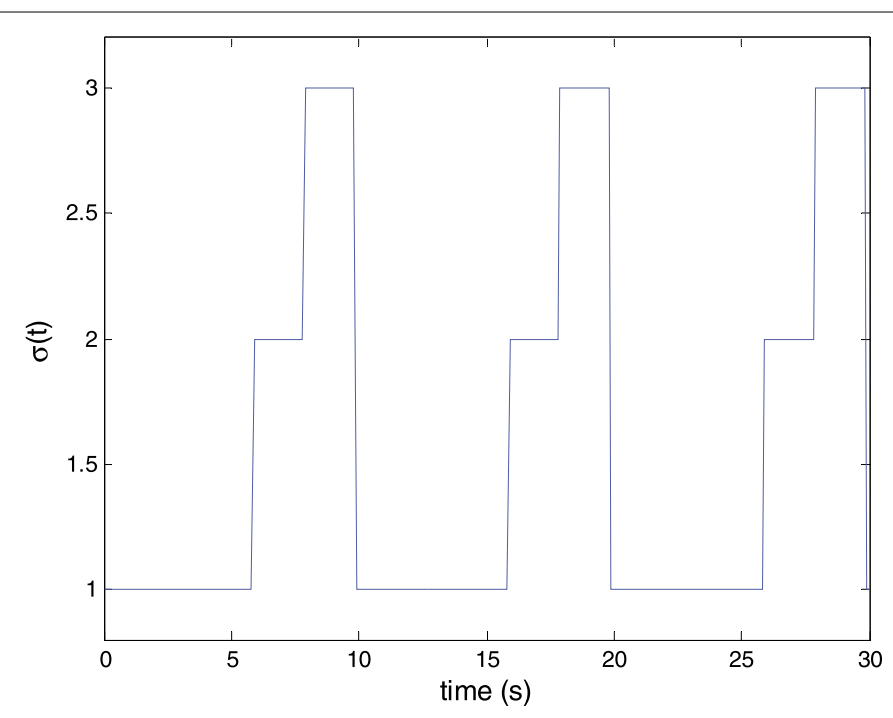

Figure 7 Switching rule (a).

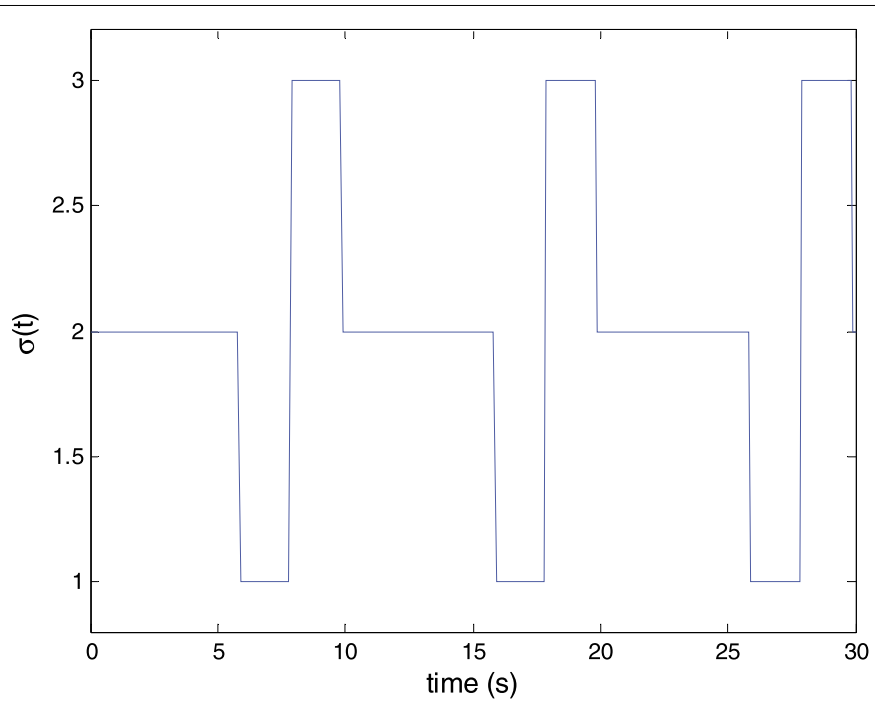

Figure 8 Switching rule (b).

Therefore, the dynamics of the system is essentially controlled by $e^{\left(-\Omega+B_{1}\right) h}, e^{\left(-\Omega+B_{2}\right) h}$, and $e^{\left(-\Omega+B_{3}\right) h}$ according to the switching rule (i.e. there are only three different acting operators). For simulation purposes consider $h=0.1$ seconds. Equations (2.2) are fulfilled for these operators with $K_{1}=1.076, K_{2}=0.905$ and $K_{3}=1$ corresponding to the expansive, strictly contractive and non-expansive (and not strictly contractive) cases (for the Euclidean distance). Now, Lemma 2.2 can be applied to establish the asymptotic stability of the origin for the switched system under different switching rules. For this purpose, assume that the switching signal is synchronized with the sampling period (i.e. the change in system's parameterization is at the same time that sampling). Let's consider these two cases: (a) the switching rule is periodic with period of 10 seconds, i.e. the switching rule is 
the periodic extension of the following signal:

$$
\sigma(t)= \begin{cases}1, & 0 \leq t<6 \\ 2, & 6 \leq t<8 \\ 3, & 8 \leq t<10\end{cases}
$$

and (b) the switching rule is periodic with period:

$$
\sigma(t)= \begin{cases}2, & 0 \leq t<6 \\ 1, & 6 \leq t<8 \\ 3, & 8 \leq t<10\end{cases}
$$

Consider now the sequence of numbers $\left\{n_{k}\right\}$ satisfying $\left(n_{k+1}-n_{k}\right) h=10$, implying that these numbers are selected when a period of the switching signal is completed. For case (a) we have $K\left(n_{k}, n_{k+1}\right)=1.076^{60} \cdot 0.905^{20} \cdot 1^{20}=10.94>1$ therefore, the switching rule (a) leads to an unstable switched system according to Lemma 2.2(iii). On the other hand, for case (b) we have $K\left(n_{k}, n_{k+1}\right)=1.076^{20} \cdot 0.905^{60} \cdot 1^{20}=0.01<1$ which leads to an asymptotically stable system according to Lemma 2.2(i). These results are corroborated by the numerical simulations shown in Figures 5 and 6 for initial condition $x_{0}=[1-1]^{T}$.

It can be appreciated in Figure 5 that the state variables absolute values enlarge with time if switching rule (a) is employed while Figure 6 shows the convergence to zero of the state variables (and the asymptotic stability of the origin) when switching rule (b) is used. Switching rules (a) and (b) are depicted for convenience in Figures 7 and 8.

Hence, the stability of the linear time-varying switched system is analyzed by just calculating the product of some constants, easing off the determination of the stability properties of the system.

\section{Competing interests}

The authors declare that they have no competing interests.

\section{Authors' contributions}

All authors contributed equally to the writing of this paper. All authors read and approved the final manuscript.

\section{Author details}

${ }^{1}$ Institute of Research and Development of Processes, University of the Basque Country, Campus of Leioa (Bizkaia), Barrio Sarriena, P.O. Box 644, Bilbao, 48940, Spain. ²Department of Telecommunications and Systems Engineering, Universitat Autònoma de Barcelona (UAB), Barcelona, 08193, Spain.

\section{Acknowledgements}

The authors are very grateful to the Spanish Government for Grant DPI2012-30651 and to the Basque Government and UPV/EHU for Grants IT378-10, SAIOTEK S-PE13UN039 and UFI 2011/07. The authors are also grateful to the referees for their suggestions.

Received: 7 September 2014 Accepted: 27 November 2014 Published: 15 Dec 2014

References

1. Berinde, V: Iterative Approximation of Fixed Points. Lecture Notes in Mathematics, vol. 1912. Springer, Heidelberg (2002)

2. Farnum, NR: A fixed point method for finding percentage points. Appl. Stat. 40(1), 123-126 (1991)

3. Antia, HM: Numerical Methods for Scientists and Engineers. Birkhäuser, Boston (2002)

4. Miñambres, JJ, De la Sen, M: Application of numerical-methods to the acceleration of the convergence of the adaptive-control algorithms: the one dimensional case. Comput. Math. Appl. 12(1), 1049-1056 (1986)

5. Soleymani, F, Sharifi, M, Sateyi, S, Khaksar Haghani, F: A class of Steffensen-type iterative methods for nonlinear systems. J. Appl. Math. 2014, Article ID 705375 (2014)

6. Samreen, M, Kamram, T, Shahzad, N: Some fixed point theorems in b-metric space endowed with graph. Abstr. Appl. Anal. 2013, Article ID 967132 (2013). doi:10.1155/2013/9067132 
7. Yao, Y, Liou, YC, Yao, JC: Convergence theorem for equilibrium problems and fixed point problems of infinite family of nonexpansive mappings. Fixed Point Theory Appl. 2007, Article ID 64363 (2007)

8. Nashine, HK, Khan, MS: An application of fixed point theorem to best approximation in locally convex space. Appl. Math. Lett. 23, 121-127 (2010). doi:10.1016/j.aml.2009.06.025

9. Yao, YH, Liou, YC, Kang, SM: An iterative approach to mixed equilibrium problems and fixed point problems. Fixed Point Theory Appl. 2013, Article ID 183 (2013). doi:10.1186/1687-1812-2013-183

10. Cho, YJ, Kadelburg, Z, Saadati, R, Shatanawi, W: Coupled fixed point theorems under weak contractions. Discrete Dyn. Nat. Soc. 2012, Article ID ID184534 (2012). doi:10.1155/2012/184534

11. Cho, SY, Li, WL, Kang, SM: Convergence analysis of an iterative algorithm for monotone operators. J. Inequal. Appl. 2013, Article ID 199 (2013). doi:10.1186/1029-242X-2013-199

12. Hussain, N, Cho, YJ: Weak contractions, common fixed points, and invariant approximations. J. Inequal. Appl. 2009, Article ID 390634 (2009)

13. Jleli, M, Karapınar, E, Samet, B: Best proximity point results for MK-proximal contractions. Abstr. Appl. Anal. 2012, Article ID 193085 (2012). doi:10.1155/2012/193085

14. Jleli, M, Samet, B: Best proximity point results for MK-proximal contractions on ordered sets. J. Fixed Point Theory Appl. (2013). doi:10.1007/s11784-013-0125-4

15. De la Sen, M, Agarwal, RP: Fixed point-type results for a class of extended cyclic self-mappings under three general weak contractive conditions of rational type. Fixed Point Theory Appl. 2011, Article ID 102 (2011). doi:10.1186/1687-1812-2011-102

16. Karapınar, E: On best proximity point of $\psi$-Geraghty contractions. Fixed Point Theory Appl. 2013, Article ID 200 (2013). doi:10.1186/1687-1812-2013-200

17. Gupta, A, Rajput, SS, Kaurav, PS: Coupled best proximity point theorem in metric spaces. Int. J. Anal. Appl. 4(2), 201-215 (2014)

18. De la Sen, M: Fixed point and best proximity theorems under two classes of integral-type contractive conditions in uniform metric spaces. Fixed Point Theory Appl. 2010, Article ID 510974 (2010)

19. Jleli, M, Karapınar, E, Samet, B: A best proximity point result in modular spaces with the Fatou property. Abstr. Appl. Anal. 2013, Article ID 329451 (2013). doi:10.1155/2013/329451

20. Pathak, HK, Shahzad, N: Some results on best proximity points for cyclic mappings. Bull. Belg. Math. Soc. Simon Stevin 20(3), 559-572 (2013)

21. De la Sen, M, Karapınar, E: Best proximity points of generalized semicyclic impulsive self-mappings: applications to impulsive differential and difference equations. Abstr. Appl. Anal. 2013, Article ID 505487 (2013). doi:10.1155/2013/505487

22. Dey, D, Kumar Laha, A, Saha, M: Approximate coincidence point of two nonlinear mappings. J. Math. 2013, Article ID 962058 (2013). doi:10.1155/2013/962058

23. Dey, D, Saha, M: Approximate fixed point of Reich operator. Acta Math. Univ. Comen. LXXXII(1), 119-123 (2013)

24. De la Sen, M, Ibeas, A: Asymptotically non-expansive self-maps and global stability with ultimate boundedness of dynamic systems. Appl. Math. Comput. 219(22), 10655-10667 (2013)

25. De la Sen, M, Agarwal, RP, Nistal, N: Non-expansive and potentially expansive properties of two modified $p$-cyclic self-maps in metric spaces. J. Nonlinear Convex Anal. 14(4), 661-686 (2013)

26. Karapınar, E: Best proximity points of Kannan type cyclic weak $\phi$-contractions in ordered metric spaces. An. Univ. 'Ovidius' Constanţa, Ser. Mat. 20(3), 51-64 (2012)

27. Karapınar, E: Best proximity points of cyclic mappings. Appl. Math. Lett. 25(11), 1761-1766 (2012)

28. Jeli, M, Karapınar, E, Samet, B: A short note on the equivalence between 'best proximity' points and 'fixed point' results. J. Inequal. Appl. 2014, Article ID 246 (2014)

29. Ashyralyev, A, Koksal, ME: Stability of a second order of accuracy difference scheme for hyperbolic equation in a Hilbert space. Discrete Dyn. Nat. Soc. 2007, Article ID 57491 (2007). doi:10.1155/2007/57491

30. Ashyralyev, A, Sharifov, YA: Optimal control problem for impulsive systems with integral boundary conditions. In: First International Conference on Analysis and Applied Mathematics (ICAAM). Book Series AIP Conference Proceedings, vol. 1470, pp. 8-11 (2012). doi:10.1063/1.4747626

31. Li, XD, Akca, H, Fu, XL: Uniform stability of impulsive infinite delay differential equations with applications to systems with integral impulsive conditions. Appl. Math. Comput. 219(14), 7329-7337 (2013)

32. Stamov, G, Akca, H, Stamova, I: Uncertain dynamic systems: analysis and applications. Abstr. Appl. Anal. 2013, Article ID 863060 (2013). doi:10.1155/2013/863060

33. Li, H, Sun, X, Karimi, HR, Niu, B: Dynamic ouput-feedback passivity control for fuzzy systems under variable sampling. Math. Probl. Eng. 2013, Article ID 767093 (2013). doi:10.1155/2013/767093

34. Xiang, Z, Liu, S, Mahmoud, MS: Robust $H_{\infty}$ reliable control for uncertain switched neutral systems with distributed delays. IMA J. Math. Control Inf. (2013). doi:2013.10.1093/imamci/dnt031

35. Tang, J, Huang, C: Impulsive control and synchronization analysis of complex dynamical networks with non-delayed and delayed coupling. Int. J. Innov. Comput. Inf. Control 9(11), 4555-4564 (2013)

36. Li, S, Xiang, Z, Karimi, HR: Stability and $L_{1}$-gain controller design for positive switched systems with mixed time-varying delays. Appl. Math. Comput. 222(1), 507-518 (2013)

37. De la Sen, M: On positivity of singular regular linear time-delay time-invariant systems subject to multiple internal and external incommensurate point delays. Appl. Math. Comput. 190(1), 382-401 (2007)

38. De la Sen, M: About the positivity of a class of hybrid dynamic linear systems. Appl. Math. Comput. 189(1), 853-868 (2007)

39. De la Sen, M: Total stability properties based on fixed point theory for a class of hybrid dynamic systems. Fixed Point Theory Appl. 2009, Article ID 826438 (2009). doi:10.1155/2009/826438

40. Marchenko, VM: Hybrid discrete-continuous systems: I. Stability and stabilizability. Differ. Equ., 48(12), 1623-1638 (2012)

41. Marchenko, VM: Observability of hybrid discrete-continuous time system. Differ. Equ. 49(11), 1389-1404 (2013)

42. Marchenko, VM: Hybrid discrete-continuous systems: II. Controllability and reachability. Differ. Equ. 49(1), 112-125 (2013) 
43. Bilbao-Guillerna, A, De la Sen, M, Ibeas, A, Alonso-Quesada, S: Robustly stable multiestimation scheme for adaptive control and identification with model reduction issues. Discrete Dyn. Nat. Soc. 2005(1), 31-67 (2005). doi:10.1155/DDNS.2005.31

44. De la Sen, M: On some structures of stabilizing control laws for linear and time-invariant systems with bounded point delays and unmensurable states. Int. J. Control 59(2), 529-541 (1994)

45. De la Sen, M, Ibeas, A: Stability results for switched linear systems with constant discrete delays. Math. Probl. Eng. 2008, Article ID 543145 (2008). doi:10.1155/2008/543145

46. De la Sen, M, Ibeas, A: On the global asymptotic stability of switched linear time-varying systems with constant point delays. Discrete Dyn. Nat. Soc. 2008, Article ID 231710 (2008). doi:10.1155/2008/231710

47. De la Sen, M, Ibeas, A, Alonso-Quesada, S: Asymptotic hyperstability of a class of linear systems under impulsive controls subject to an integral Popovian constraint. Abstr. Appl. Anal. 2013, Article ID 382762 (2013). doi:10.1155/2013/382762

48. Niamsup, P, Rojsiraphisa, T, Rajchakit, M: Robust stability and stabilization of uncertain switched discrete-time systems. Adv. Differ. Equ. 2012, Article ID 134 (2012). doi:10.1186/1687-1847-2012-134

49. Niamsup, P, Rajchakit, G: New results on robust stability and stabilization of linear discrete-time stochastic systems with convex polytopic uncertainties. J. Appl. Math. 2013, Article ID 368259 (2013). doi:10.1155/2013/368259

50. Darwish, MA, Henderson, J: Existence and asymptotic stability of solutions of a perturbed quadratic fractional integral equation. Fract. Calc. Appl. Anal. 12(1), 71-86 (2009)

51. De la Sen, M: Robust stability of a class of linear time-varying systems. IMA J. Math. Control Inf. 19(4), 399-418 (2002)

52. Tsakalis, KS, loannou, PA: Linear Time-Varying Systems. Prentice Hall, New York (1993)

10.1186/1029-242X-2014-498

Cite this article as: De la Sen and Ibeas: Properties of convergence of a class of iterative processes generated by sequences of self-mappings with applications to switched dynamic systems. Journal of Inequalities and Applications 2014, 2014:498

\section{Submit your manuscript to a SpringerOpen ${ }^{\ominus}$ journal and benefit from:}

- Convenient online submission

- Rigorous peer review

- Immediate publication on acceptance

- Open access: articles freely available online

- High visibility within the field

- Retaining the copyright to your article 